\title{
Automatic Time Series Forecasting with Ata Method in R: ATAforecasting Package
}

\author{
by Ali Sabri Taylan, Güçkan Yapar and Hanife Taylan Selamlar
}

\begin{abstract}
Ata method is a new univariate time series forecasting method that provides innovative solutions to issues faced during the initialization and optimization stages of existing methods. The Ata method's forecasting performance is superior to existing methods in terms of easy implementation and accurate forecasting. It can be applied to non-seasonal or deseasonalized time series, where the deseasonalization can be performed via any preferred decomposition method. The R package ATAforecasting was developed as a comprehensive toolkit for automatic time series forecasting. It focuses on modeling all types of time series components with any preferred Ata methods and handling seasonality patterns by utilizing some popular decomposition techniques. The ATAforecasting package allows researchers to model seasonality with STL, STLplus, TBATS, stR, and TRAMO/SEATS, and power family transformation and analyze the any time series with a simple Ata method and additive, multiplicative, damped trend the Ata methods and level fixed Ata trended methods. It offers functions for researchers and data analysts to model any type of time series data sets without requiring specialization. However, an expert user may use the functions that can model all possible time series behaviors. The package also incorporates types of model specifications and their graphs, uses different accuracy measures that surely increase the Ata method's performance.
\end{abstract}

\section{Introduction}

Ata method (Cetin and Yavuz, 2020; Yilmaz et al., 2019; Yapar et al., 2019; Yapar, 2018; Yapar et al., $2018,2017)$ is a new univariate time series forecasting method which provides innovative solutions to issues faced during the initialization and optimization stages of existing methods. ATAforecasting performance is superior to existing methods both in terms of easy implementation and accurate forecasting. It can be applied to non-seasonal or deseasonalized time series, where the deseasonalization can be performed via any preferred decomposition method. This methodology performed extremely well on the M3 and M4-Competition data.

The original exponential smoothing has accomplished well in a wide range of practical researches, and it is well built as a precise and optimal forecasting method. Nonetheless, two essential difficulties are to choose the smoothing constant and starting value in exponential smoothing theory. The Ata method suggests an alternative method for smoothing constant and initial value. The Ata method places more emphasis than the classical method on most recent activities. The forecasting error is compared to the error in forecasts obtained by the original model.

Exponential smoothing (ES) is not the only model. In fact, a family of models. ES models suppose that a time series has four components: seasonality, trend, level, and remainder. Bergmeir et al. (2016) recommended the bootstrap aggregation of ES methods. The bootstrap aggregation employs a Box-Cox transformation afterwards an a seasonal trend decomposition based on LOESS (LOcally Estimated Scatter-plot Smoother) (STL) to segregate the time series sub three part: remainder, seasonal and trend. The remainder is then bootstrapped via a moving block, and a new data is gathered via this bootstrapped residual part. Thereafter, an ensemble of ES models is calculated with the bootstrapped series.

Incorporating other types of model specifications and using different accuracy measures will surely increase the Ata method's performance. Like other approaches, the method can also benefit from certain transformations and decompositions of other types of more involved combinations, outlier detection, and other more complicated model selection strategies. The fact that these simple selection and combination strategies can perform better than existing methods is fascinating, and this further strengthens the idea that simplicity is indeed a prerequisite for forecasting accuracy. 


\begin{tabular}{ll}
\hline \hline Function & Description \\
\hline ATA & Time series analysis and forecasting using the Ata method. \\
ATA.Core & The core algorithm of the Ata method. \\
ATA.Forecast & Produces forecasts from the output of ATA function. \\
ATA.Accuracy & Computes fitting and forecasting accuracy measures. \\
ATA.Transform & Computes transformed data using power transformation techniques. \\
ATA.BackTransform & Computes back transformed data using power transformation techniques. \\
ATA.BoxCoxAttr & Assigns attributes set for unit root and seasonality tests. \\
ATA.Seasonality & Tests seasonality. \\
ATA.Decomposition & Decomposes a time series into seasonal, trend, and irregular. \\
ATA.SeasAttr & Assigns attributes set for unit root and seasonality tests. \\
ATA.Plot & Specialized plot function of the output of ATA function. \\
ATA.Print & Specialized print screen function of the output of ATA function. \\
ATA.CI & Confidence interval function for the Ata method forecasts. \\
\hline \hline
\end{tabular}

Table 1: A summary of the functions available in the ATAforecasting package.

Several decomposition techniques are used before applying the Ata method for selecting an optimized model. The high performance of these combined models is indicated with an empirical practice. The Ata method is analyzed in contrast practically with the most well-known forecasting techniques based on ES and ARIMA in accordance with its predictive performance on the M3 (Makridakis and Hibon, 2000) and M4-Competitions (Makridakis et al., 2018) data set and is illustrated to outperform its contestants.

Over the past few years, the preliminary research on ES (Brown, 1959; Pegels, 1969; Gardner and McKenzie, 1985) expanded to an approach based on a model so that there are 30 potential ES models for various types of trend, seasonality, and errors. The well-known of these are the simple ES, Holt's linear trend model, and Holt-Winter's model. Then, Gardner and McKenzie (1985) proposed damped trend model to help deal with overtrending. The reputation and universality of ES can also be attributed to its proven record against more sophisticated techniques (Makridakis et al., 1984; Makridakis and Hibon, 2000; Koning et al., 2005). The forecast package (Hyndman et al., 2020) in the programming language $\mathrm{R}$ (R Core Team, 2016) means that a fully automated software for fitting ETS models is available. These have led to a broadly appropriate ES modelling background, and with the use of latterly developed software packages, these ES models handle seasonality, trend, and other attributes of series without any human intervention (Hyndman et al., 2002, 2008; Hyndman and Athanasopoulos, 2019).

The Theta method (Assimakopoulos and Nikolopoulos, 2000) was introduced as a new univariate forecasting method which is similar to a simple ES model with drift, and its performance in terms of forecasting accuracy was prominent in M3-Competition. As confirmed once again in Assimakopoulos and Nikolopoulos (2000), it is well known that combining forecasts (Bates and Granger, 1969; Clemen, 1989) under certain circumstances improves forecasting accuracy (Armstrong, 1989, 2001; Makridakis and Winkler, 1983; Makridakis et al., 1982). Due to this, the research focuses on transformations, decompositions, rules, and combinations of ES and ARIMA (a few examples are (Clemen, 1989; Cleveland et al., 1990; Adya et al., 2000) to improve the forecasting performance rather than suggesting new forecasting methods.

Several other studies that are based on automatic forecasting procedures exist. Particularly for seasonal time series, the forecast package offers the TBATS model (Livera et al., 2011). TBATS uses a parsimonious trigonometric representation of seasonality instead of conventional seasonal indices and also incorporates ARMA errors. In addition, the function also automatically performs Box-Cox transformation of the time series if necessary.

This study introduces ATAforecasting (available from the Comprehensive R Archive Net- work at https://cran.r-project.org/package=ATAforecasting), a software application for R which performs a novel decomposition and power transformation-based approaches to time series forecasting using Ata method without any academic expertise. To sum up, the ATAforecasting package (Taylan et al., 2021a) provides a novel $\mathrm{R}$ interface for researchers interested in automatic time series analysis and students and academics who teach courses related to univariate time series analysis topics. There are main 13 functions available in the ATAforecasting package; see Table 1. We are going to describe all of them as we go on to explain the theoretical procedure. The rest of the paper is organized as follows. Section 2 presents a novel forecasting approach using the Ata method, gives an overview of the main estimation methods of the Ata method, and provides some technical details about the ATAforecasting package. Section 3 illustrates M-forecasting Competition dataset examples showing the package's functionality. Section 4 contains some concluding remarks. 


\section{Methodology}

The objective of this study is to introduce a new decomposition-based approach to time series forecasting with the Ata method to provide the automation and the optimization of the Ata method which is an innovative and accurate univariate time series analysis method without any expertise of the R program. Specifically, we propose an analytical methodology of time series method with ATAforecasting $\mathrm{R}$ package, as it combines several stationarities and seasonality tests, Box-Cox transformations, seasonal decomposition techniques with the Ata method. We merge the various preceding concepts to attain a robust and broadly practicable automatic forecasting algorithm. The methodology involves 2 alternative algorithms with 6 steps as described and summarized below:

\section{First Algorithm in Figure 1a}

- Step 1 Transformation Transformation for stabilizing the variance of a time series if necessary;

There are many power transformation methods available to stabilizing linearity and variance. In this paper, logarithm, logarithm with shift parameter, Box-Cox, Box-Cox with shift parameter, Modulus, Bickel-Doksum, Dual, Yeo-Johnson, generalized logarithm (glog), and glog with power function (gpower) methods are able to applied.

- Step 2 Seasonality Test

Identify and correct for seasonality in time series;

There are several methods to detect stationarity and seasonality in time series. In this package, Augmented Dickey-Fuller (ADF), Phillips and Perron (PP), Kwiatkowski, Phillips, Schmidt, and Shin (KPSS) unit root tests are adopted for stationarity. Autocorrelation function (ACF), CanovaHansen (CH), Hylleberg-Engle-Granger-Yoo (HEGY), Osborn, Chui, Smith, and Birchenhall (OCSB) seasonal unit root tests are adopted for seasonality.

- Step 3 Decomposition

Decompose the series into three components: trend, seasonal, and remainder;

There are a few techniques to decompose time series. In this package, classical decomposition (decompose - stats package (R Core Team, 2016)), the Seasonal-Trend decomposition using LOESS (STL - stats package (R Core Team, 2016)), an enhanced of STL method (stlplus package (Hafen, 2016)), Trigonometric Seasonal Box-Cox Transformation-ARMA residuals-Trend and Seasonality (TBATS - forecast package (Hyndman et al., 2020)), Seasonal-Trend Decomposition Procedure Based on Regression (stR package (Dokumentov and Hyndman, 2018)) are adopted.

- Step 4 ATA Forecasting

Apply ATA forecasting method to generate forecasts for the time series;

Although there are many forecasting techniques available to perform (e.g., ETS, ARIMA, Theta, etc.), the Ata forecasting method is used. Ata method is an innovative new forecasting technique where the forms of the models are similar to exponential smoothing models. Still, the smoothing parameters depending on the sample size are optimized in a discrete space. Initialization is easier as it is done simultaneously when the parameters are optimized and less influential since the weights assigned to initial values approach zero quickly.

- Step 5 Selection and Aggregation The model fits all possible ATA models to the data, then chooses the best model using the accuracy measures. Aggregate the best selected ATA forecast model for trend + remainder components and seasonal component to generate the final result. The final outcome is calculated from the forecasts from the single ATA models.

- Step 6 Inverse Transformation

\section{Second Algorithm in Figure $1 \mathrm{~b}$}

- Step 1 Seasonality Test

- Step 2 Decomposition

- Step 3 Transformation

- Step 4 ATA Forecasting

- Step 5 Selection

- Step 6 Inverse Transformation and Aggregation

To summarize, the ATAforecasting procedure is given in Figure 1. As default, initially, the selected power family transformation is implemented, and the series are decomposed into the seasonal part and trend + remainder part, using the selected decomposition technique. Then, the Ata method is applied to the trend + remainder part. The components are added together again, and the selected power family transformation is inverted. 


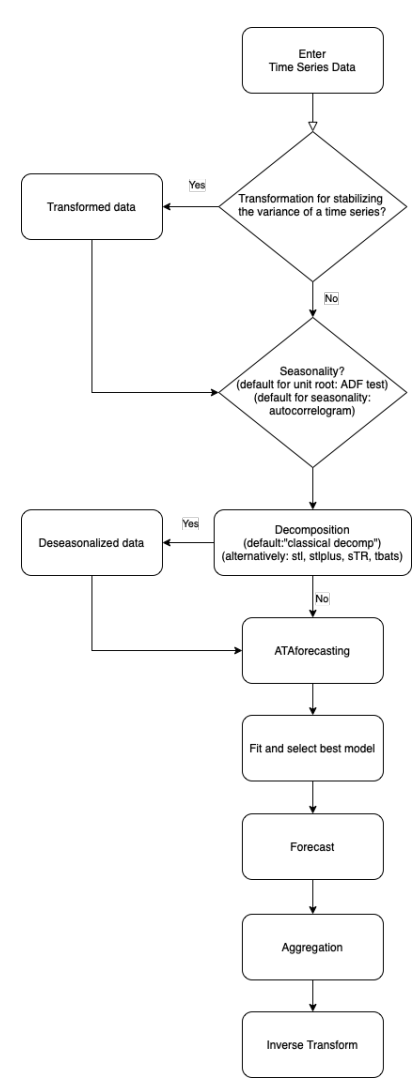

(a) First Algorithm

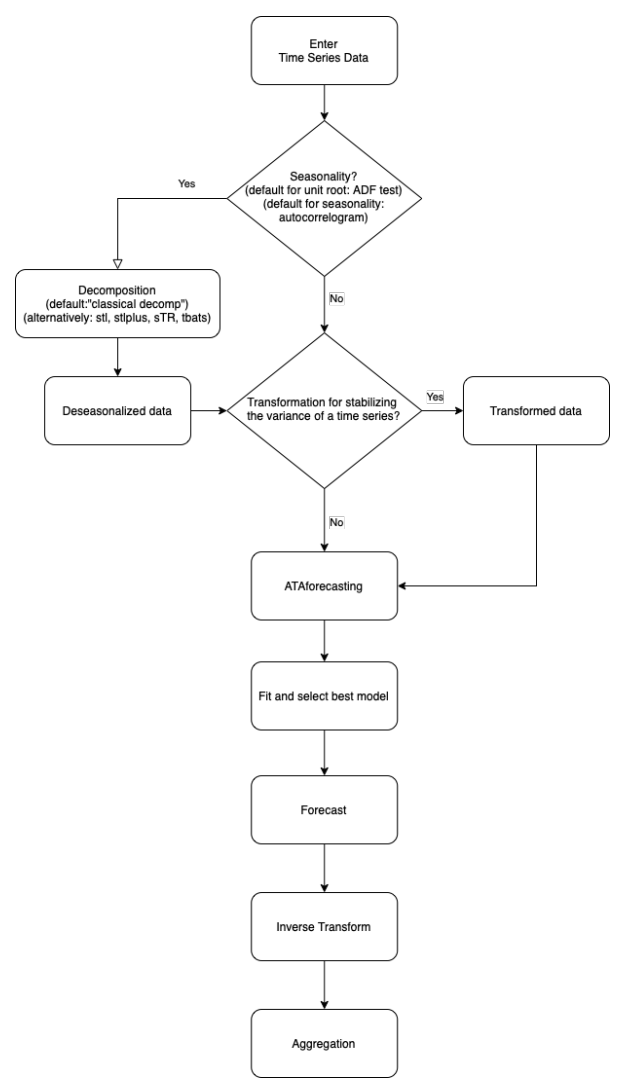

(b) Second Algorithm

Figure 1: Algorithms of ATAforecasting procedure.

\section{Power transformation family for ATAforecasting package}

Traditional statistical procedures often assume that the data is homoscedastic and normally distributed. Despite its apparent restrictions, the logarithmic transformation has been used mostly when data violates these assumptions. The purpose of a particular transformation for better fitting is additivity, convergence to normality, stationarity, linearity, reduction of skewness, stabilizing variance. These purposes, which may even be inconsistent, are quite significant as it is just under such assumptions that particular statistical methods are relevant. Generally, logarithmic transformations almost stabilize the variance for time series consisting of large values. Some of the problems that arise when implementing a specific transformation are argued in different settings by Sakia (1992), Staniswalis et al. (1993), Quiroz et al. (1996), Yeo and Johnson (2000), Chen et al. (2002), Mu and He (2007), Horowitz (2009) and Meintanis and Stupfler (2015). There are many proposed methods of transformation and a large amount of research in the literature. Sakia (1992) provided a detailed and extensive review of the Box-Cox (Box and Cox (1964)) and some alternative versions. Different methodology recommended for choosing the appropriate value of transformation parameters based on maximizing the likelihood function (Box and Cox (1964)) or alternatively, Kullback-Leibler information-based method (Hernandez and Johnson, 1980), robust adaptive method (Carroll, 1980) and a method based on Kendall's rank correlation, (Han, 1987).

A chiefly used algorithm of the Box-Cox family is the logarithm transformation, which is convenient for multiplicative process data. Moreover, the asymptotic variance of a time series can be stabilized by the log-transformation. A shift parameter was additionally proposed to apply the log transformations more responsive and handy. The parameterizations of the shift parameter depend on knowledge of the data e.g., data range, data distribution, so user intervention is usually required. ATAforecasting package automates the selection of shift parameter, which is an important contribution of automatic times series forecasting.

Selected transformation functions included in the ATAforecasting R package provide the applicability of different types of transformation techniques for the data to which the Ata method will be applied. The ATA. Transform function works with many different types of inputs. Many power transformation methods are available to stabilize linearity and variance. In this package, power transformation family is consist of "Box-Cox", "Sqrt", "Reciprocal", "Log", "NegLog", "Modulus", "Bickel-Doksum", "Manly", "Dual", "Yeo-Johnson", "GPower", "GLog". If the transformation process 
needs a shift parameter, ATA. Transform will calculate the required shift parameter automatically.

- Log Transformation with Shift,

- Box-Cox Transformation with Shift (Box and Cox, 1964),

- GLog Transformation (Durbin et al., 2002),

- NegLog Transformation (Whittaker et al., 2005),

- Reciprocal Transformation (Tukey, 1977),

- Bickel-Doksum Transformation (Bickel and Doksum, 1982),

- Yeo-Johnson Transformation (Yeo and Johnson, 2000),

- Modulus Transformation (John and Draper, 1980),

- Dual Power Transformation (Yang, 2005),

- GPower Transformation (Kelmansky et al., 2013),

The ATA.BoxCoxAttr function

Since the main ATA function and ATA. Transform are designed by some attributes of Box-Cox power transformation family, we provide the user with the function ATA.BoxCoxAttr.

The R function ATA.BoxCoxAttr can be utilized with the following code,

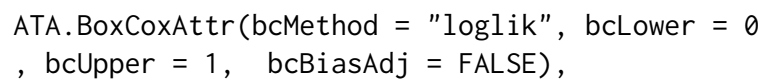

and makes use of four parameters. These are

- bcMethod: Choose method to be used in calculating lambda. "loglik" is default. Other method is "guerrero" (Guerrero, 1993).

- bcLower: Lower limit for possible lambda values. The lower value is limited by -5 . Default value is 0 .

- bcUpper: Upper limit for possible lambda values. The upper value is limited by 5 . Default value is 1 .

- bcBiasAdj: Use adjusted back-transformed mean for Box-Cox transformations. If transformed data is used to produce forecasts and fitted values, a regular back transformation will result in median forecasts. If $b c B i a s A d j$ is TRUE, an adjustment will be made to produce mean forecasts and fitted values. If $b c B i a s A d j=T R U E$, optional parameter foar required. fvar can either be the forecast variance or a list containing the interval level and the corresponding upper and lower intervals. Default value of foar is NULL and it can't be changed.

The ATA. Transform function

The main function of power transformations, the ATA. Transform, can be called with

ATA.Transform(X, tMethod = "Box_Cox", tLambda

, tShift $=0$, bcMethod $=$ "loglik", bcLower $=0$, bcUpper $=1$ )

and it makes use of seven parameters and returns three outputs. The inputs are

- $X$ : a numeric vector or time series of class ts or msts for in-sample.

- tMethod: Power transformation family is consist of "Box_Cox", "Sqrt", "Reciprocal", "Log", "NegLog", "Modulus", "BickelDoksum", "Manly", "Dual", "YeoJohnson", "GPower", "GLog" in ATAforecasting package. If the transformation process needs shift parameter, ATA. Transform will calculate the required shift parameter automatically.

- tLambda: Box-Cox power transformation family parameter. If NULL, data transformed before model is estimated.

- tShift: Box-Cox power transformation family shifting parameter. If NULL, data transformed before model is estimated.

- bcMethod: Choose method to be used in calculating lambda. "loglik" is default. Other method is "guerrero" (Guerrero, 1993).

- bcLower: Lower limit for possible lambda values. The lower value is limited by -5 . Default value is 0 .

- bcUpper: Upper limit for possible lambda values. The upper value is limited by 5. Default value is 5 . 
The outputs are

- $\operatorname{trfmX}$ : Transformed data.

- tLambda: Box-Cox power transformation family parameter.

- tShift : Box-Cox power transformation family shifting parameter.

To apply this algorithm to an example in the tsibbledata package "aus_retail", monthly retail turnover (in million AUD) in Australian states from April 1982 to December 2018, we use the following commands.

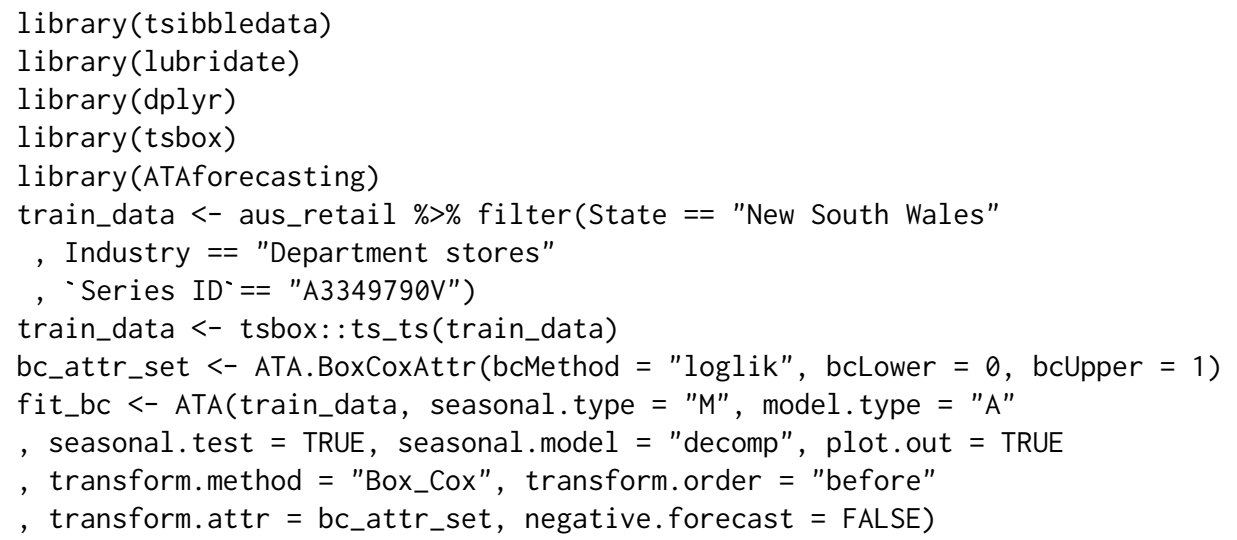

\section{Seasonality for ATAforecasting package}

Seasonality is a well-known phenomenon observed in many economic time series. Seasonal decomposition, which is the first stage of a time series modeling, is also the first stage of the Ata method. The performance of the Ata method has been improved after the seasonal decomposition.

Specifically, our proposed methodology to identify seasonality in time series is as follows. After or before implementing a Box-Cox transformation (if necessary) to the data, the data is decomposed into remainder, seasonal, and trend components. The trend and remainder components are then forecasted via the Ata method, the seasonal component is added back in, and the Box-Cox transformation is inverted. Then, point forecasts are calculated using each of the different models, and/or the resulting forecasts are able to be combined.

In previous studies, the classical decomposition method is much used after the seasonality test. With this package, stl, stlplus, tbats, and stR decomposition techniques are also available choices by the ATAforecasting package, which can be chosen with only one or multiple.

Seasonality for ATAforecasting package enables estimating all of the below components and specifications. The main functions of seasonality in the package are the following

- ATA.SeasAttr(),

- ATA.Seasonality(),

- ATA.Decomposition().

Three seasonality diagnostics methods are able to be applied in the package.

- Unit Root Tests,

- Seasonal Unit Root Tests,

- Seasonal Decomposition.

\section{The ATA. SeasAttr function}

This function is a class of seasonality tests using corrgram. test from ATAforecasting package, ndiffs and nsdiffs functions from forecast package. Also, ndiffs and nsdiffs functions have been modified according to different unit root testing packages. Please review manual and vignette documents of the latest forecast package. According to forecast package, ndiffs and nsdiffs functions estimate the number of differences requisite to ensure stationary of a given time series.

ndiffs employs unit root tests to define required number of differences for time series to be ensured trend stationary. nsdiffs employs seasonal unit root tests to define required number of seasonal differences for time series to be ensured trend stationary.

The ATA. SeasAttr function works with many different types of inputs. The inputs are below. 
- corrgram.tcrit: $t$-value for periodogram seasonality test.

- uroot.test: Type of unit root test before all type seasonality test. Possible values are "adf", "pp", and "kpss".

- suroot.test: Type of seasonal unit root test to use. Possible values are "correlogram", "sea", "hegy", "ch", and "ocsb".

- suroot.uroot: If TRUE, unit root test for stationary before seasonal unit root test is allowed.

- uroot.type: Specification of the deterministic component in the regression for unit root test. Possible values are "level" and "trend".

- uroot.alpha: Significant level of the unit root test, possible values range from 0.01 to 0.1 .

- suroot.alpha: Significant level of the seasonal unit root test, possible values range from 0.01 to 0.1 .

- uroot.maxd: Maximum number of nonseasonal differences allowed.

- suroot.maxD: Maximum number of seasonal differences allowed.

- suroot.m: Deprecated. Length of seasonal period: frequency of data for nsdiffs.

- uroot.pkg: Using ucra or tseries packages for unit root test. The default value is ucra.

- multi.period: Selection type of multiseasonal period. min or max function for selection.

- x13.estimate.maxiter Maximum: iteration for X13ARIMA/SEATS estimation.

- x13.estimate.tol: Convergence tolerence for X13ARIMA/SEATS estimation.

- x11.estimate.maxiter Maximum: iteration for X11 estimation.

- x11.estimate.tol: Convergence tolerence for X11 estimation.

\section{Unit root tests}

Unit root tests for stationarity have compatibility in almost every practical time series analysis. Choosing which unit root procedure to employ is an issue of active interest. In this study, we implement the three widely used unit root tests. In accordance with past research, the selected unit root tests occasionally disagree in choosing the convenient order of integration for a given data. The following literature shows the basic features of unit root tests. Users who demand details should consult the original resources and standard references (see, for example, (Davidson and MacKinnon, 1993; Hamilton, 1994; Hayashi, 2000)).

In the ATAforecasting package, the following unit roots methods are able to be applied.

- Augmented Dickey-Fuller Test (Dickey and Fuller, 1979; Said and Dickey, 1984)

- Phillips and Perron Test (Phillips and Perron, 1988)

- Kwiatkowski, Phillips, Schmidt, and Shin Test (Kwiatkowski et al., 1992)

\section{The ATA. SeasAttr function for unit root tests}

Since the main ATA function and ATA. Seasonality are designed by some attributes of unit root tests, we provide the user with the function ATA. SeasAttr.

For the main function ATA, the attributes of unit root test can be accessed with

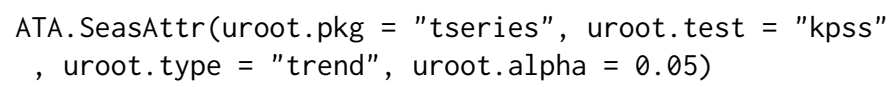

The following code uses the unit root test approach to search trend component before the seasonality test of the data in the context of the Ata method.

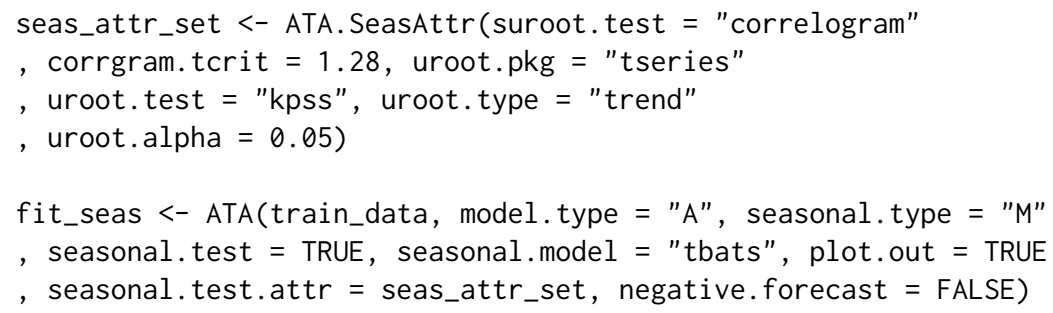




\section{Seasonality tests}

There are numerous studies relating seasonality and unit root. One of these studies uses autocorrelogram. Autocorrelation function and partial autocorrelation function are useful qualitative tools to estimate the existence of autocorrelation at individual lags. The Ljung-Box Q-test is a more quantitative method to test autocorrelation at multiple lags jointly. Other techniques generally use unit root tests. Hylleberg et al. (1990) improved unit root tests in linear time series regarding seasonality and studied with different models, including different combinations of seasonal, trend, remainder, and constant parts. Their purpose is to improve a testing process that will determine what class of seasonality is accountable for the seasonality in a time series process. There exist more studies for testing seasonal unit roots, such as Ljung and Box (1978), Dickey et al. (1984), Osborn et al. (1988), and Wang et al. (2006).

In the ATAforecasting package, the following methods are able to applied.

- Autocorrelogram (Ljung and Box, 1978)

- Canova-Hansen (CH) Test (Canova and Hansen, 1995)

- Hylleberg, Engle, Granger E Yoo (HEGY) Test (Hylleberg et al., 1990)

- Osborn, Chui, Smith, Birchenhall (OCSB)Test (Osborn et al., 1988)

- Seasonal Strength Measure (Wang et al., 2006)

\section{The ATA. SeasAttr function for seasonal unit root test}

Since the main ATA function and ATA. Seasonality are designed by some attributes of seasonality tests, we provide the user with the function ATA. SeasAttr.

For the main function ATA, the attributes of seasonality test can be accessed with

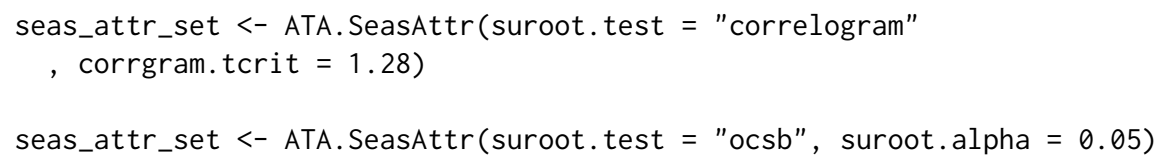

An example of the seasonality test's call is the following

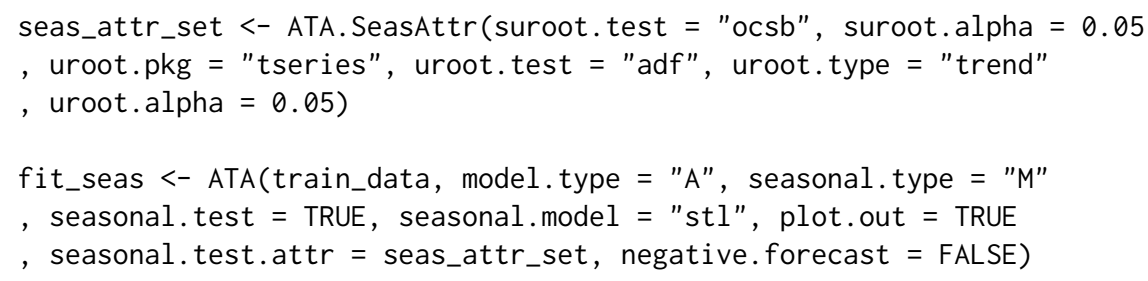

The ATA. Seasonality function for Seasonality Tests

The ATAforecasting's seasonality diagnostics described before in this paper are implemented into a function named ATA. Seasonality that can calculate all of them respectively. The function syntax is

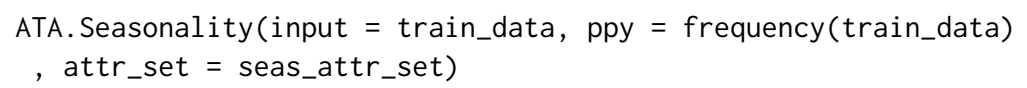

The ATA. Seasonality function works with many different types of inputs. The inputs are below.

- input: The data.

- ppy: Frequency of the data.

- attr_set: Assign from ATA. SeasAttr function. Attributes set for the unit root, seasonality tests.

Here is an another simple example, applying ATA. SeasAttr and ATA. Seasonali ty to the M3 data:

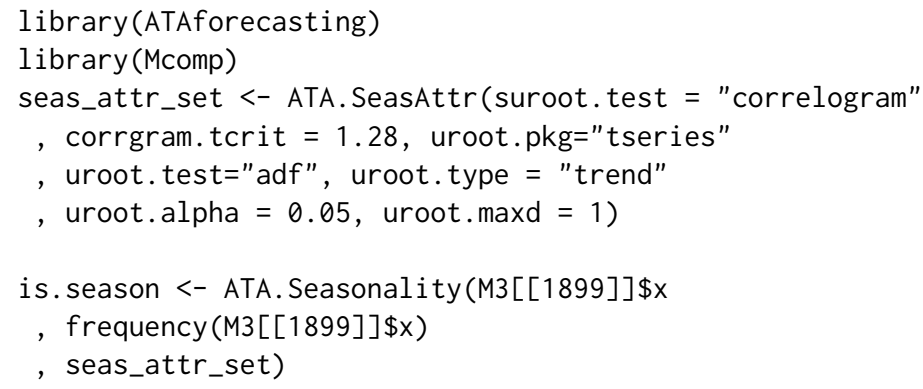




\section{Seasonal decomposition}

A substantial aim in time series analysis is the decomposition of a time series into latent parts that can be incorporated with dissimilar versions of temporal variations. Persons (1919) was the first to state the assumptions of latent parts particularly. Persons indicated that time series was constituted of four types of fluctuations (Dagum and Bianconcini, 2016): residual variations, seasonal movement, secular trend, and cyclical movements. Further, research in that direction included Copeland (1915) and Persons (1919), who introduced for extracting the seasonal component until Macaulay (1931) suggested a technique which turned into "classical" in the log run.

Macaulay (1931) developed a computer program which is significantly simplified the calculations (Shiskin, 1957). Extensively used various techniques and features which have such as ARIMA extensions, regressors, calendar effects, robustness, and extensive diagnostics in literature are X-11 (Shiskin et al., 1967), X-11-ARIMA (Dagum, 1988), X-12-ARIMA (Findley et al., 1998) and X-13ARIMASEATS (Monsell et al., 2003; Findley, 2005; Monsell, 2007). X-13ARIMA-SEATS contains a type of the TRAMO/SEATS procedure which was improved by the Bank of Spain for seasonal adjustment.

Cleveland et al. (1990) recommended a different approach and developed STL (Seasonal Trend decomposition using LOESS) based on local regression familiar as moving regression which is a generalization of moving average and polynomial regression. LOESS is a connected nonparametric method that assembles multiple regression models in a metamodel based on the k-nearest neighbor. Burman (1980) discussed plenty of seasonal adjustment techniques and remarked that all but one were ad hoc techniques. Since this study, several model-based methods for seasonal decomposition have been evolved, including the TRAMO/SEATS procedure, assorted structural time series models (Harvey, 1990; Commandeur et al., 2011) and the BATS and TBATS models of Livera et al. (2011).

Conventionally, the four variations suppose to be mutually independent of one another and signify by means of an additive decomposition model. If there is dependency among the hidden parts, this relation is signified via a multiplicative decomposition model. In some cases, combined additive and multiplicative models can be employed. See Dagum and Bianconcini (2016) for further details.

\section{The ATA. Decomposition function for seasonality}

Automatic seasonal decomposition for the ATA method is called ATA. Decomposition function in the ATAforecasting package. The function returns seasonally adjusted data constructed by removing the seasonal component. The methodology is fully automatic. The ATA. Decomposition function works with many different types of inputs. The inputs are below.

- input: It must be ts, msts, or numeric object. If it is a numeric object, findPeriod must be 1, 2, 3 or 4 . If it is a msts object, findPeriod must be 3 or 4 .

- s.model: A string identifying method for seasonal decomposition. If NULL, "decomp" method is default. c("none", "decomp", "stl", "stlplus", "tbats", "stR") phrases of methods denote.

- none: seasonal decomposition is not required.

- decomp: classical seasonal decomposition. If decomp, the stats package will be used.

- stl: seasonal-trend decomposition procedure based on LOESS developed by Cleveland et al. (1990). If $s t l$, the stats and forecast packages will be used. Multiple seasonal periods are allowed.

- stlplus: seasonal-trend decomposition procedure based on LOESS developed by Cleveland et al. (1990). If stlplus, the stlplus package will be used.

- tbats: exponential smoothing state space model with Box-Cox transformation, ARMA errors, trend, and seasonal components as described in Livera et al. (2011). Parallel processing is used by default to speed up the computations. If $t b a t s$, the forecast package will be used. Multiple seasonal periods are allowed.

- stR: seasonal-trend decomposition procedure based on the regression developed by Dokumentov and Hyndman (2015). If $s t R$, the stR package will be used. Multiple seasonal periods are allowed.

- x13: seasonal-trend decomposition procedure based on X13ARIMA/SEATS. If $x 13$, the seasonal package will be used.

- x11: seasonal-trend decomposition procedure based on X11. If $x 11$, the seasonal package will be used.

- s.type: A one-character string identifying method for the seasonal component framework. If NULL, "M" is default. The letter "A" for additive model, the letter "M" for multiplicative model.

- s.frequency: Value(s) of seasonal periodicity. If s.frequency is not integer, $X$ must be msts time series object. $\mathrm{c}(\mathrm{s} 1, \mathrm{~s} 2, \mathrm{~s} 3, \ldots)$ for multiple period. If $X$ has multiple periodicity, "tbats" or "stR" 
seasonal model have to be selected. For example, period of the input data, which have one seasonal pattern $\rightarrow 12$ for monthly / 4 for quarterly / 7 for daily / 5 for business days. Periods of the input data which have complex/multiple seasonal patterns $\rightarrow c(7,354.37,365.25)$.

- seas_attr_set: Assign from ATA.SeasAttr function. Attributes set for unit root, seasonality tests.

ATA. Decomposition function returns four outputs. The outputs are below.

- AdjustedX : Deseasonalized data.

- SeasIndex : Particular seasonality data given cycle/frequency.

- SeasActual : Seasonality given original data.

- SeasType : Seasonal decomposition technique.

As an example, let us compute seasonal decomposition on the real life tsibbledata dataset shown in the following seven figures (Figures 2, 3, 4, 5, 6, and 7).

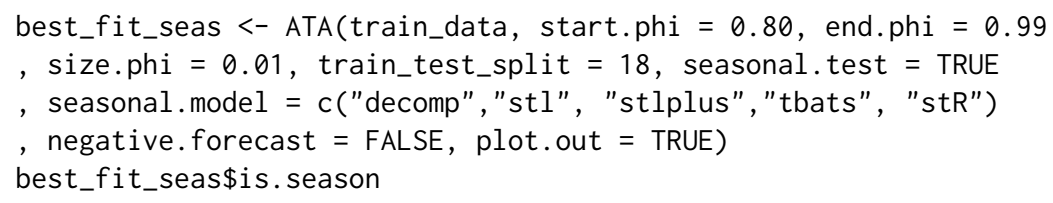



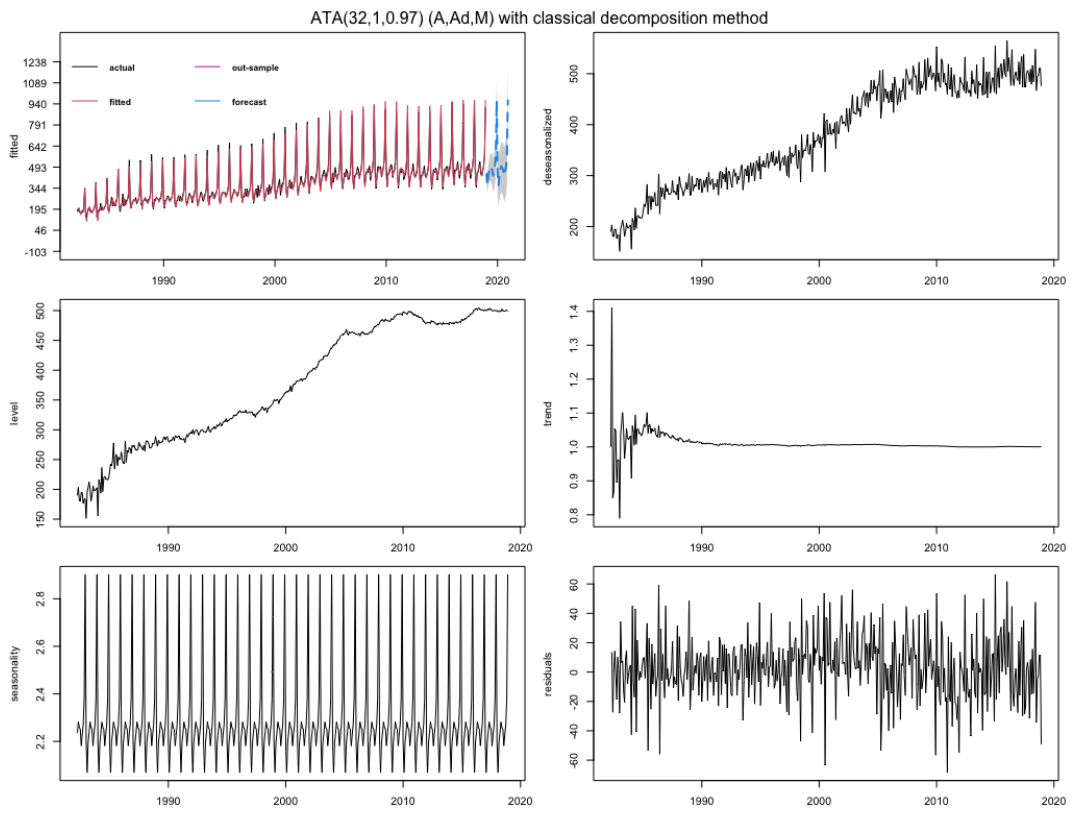

Figure 2: Best forecasts for the Australian Retail Turnover using ATA seasonal trended methods.
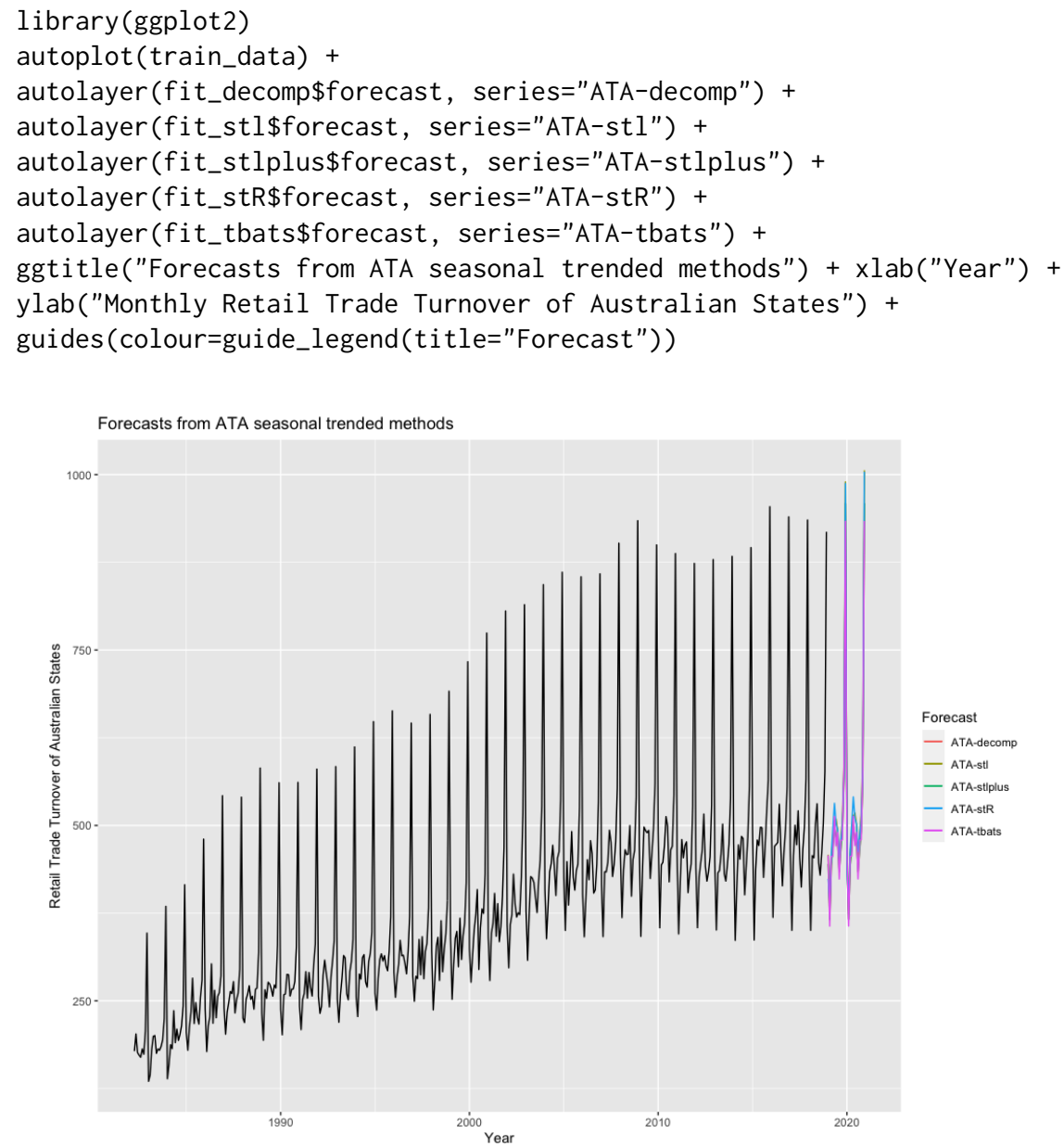

Figure 3: Forecasts from ATA seasonal trended methods. 
There are five different techniques for seasonal decomposition in the package. We use the following techniques

- Classical Decomposition: The classical method of time series decomposition originated in the 1920s and was widely used until the 1950s.

fit_decomp <- ATA(train_data, seasonal.test = TRUE

, seasonal.model $=$ "decomp", negative.forecast $=$ FALSE)
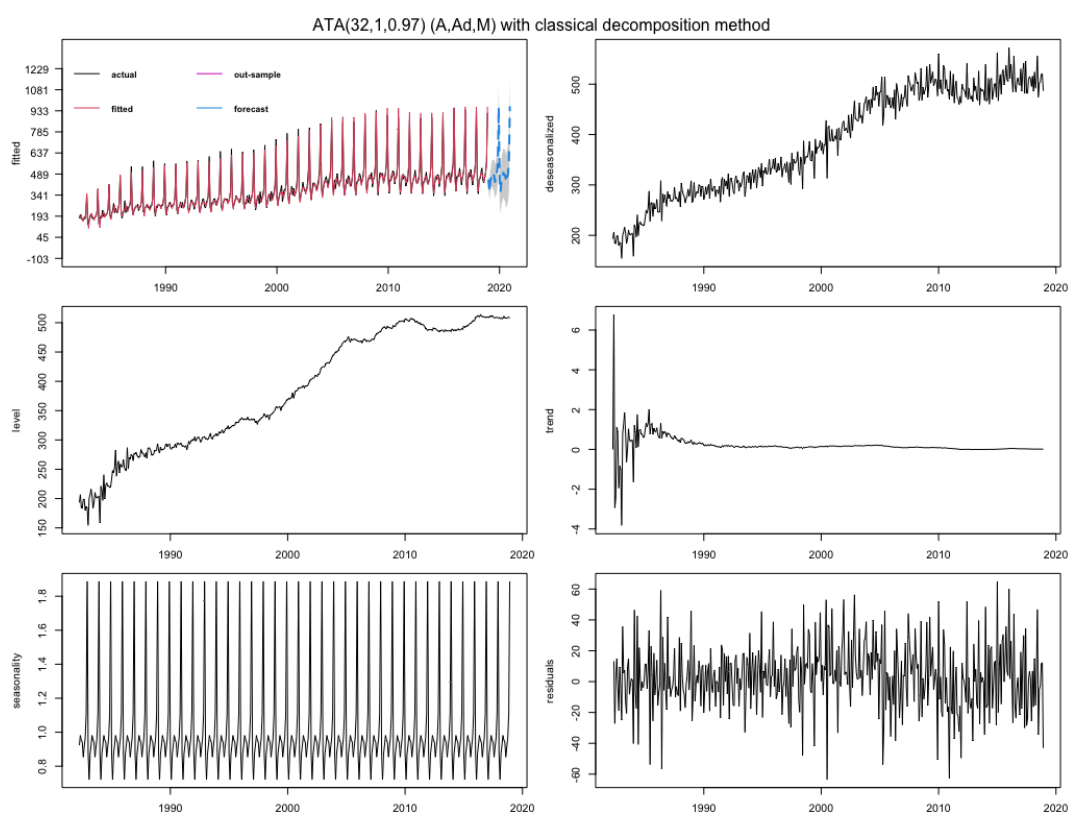

Figure 4: The Ata method with classical decomposition.

- STL Decomposition (Cleveland et al., 1990):

fit_stl <- ATA(train_data, model.type = "A", seasonal. type = "M" , seasonal.test $=$ TRUE, seasonal. model $=$ "stl", negative. forecast $=$ FALSE)
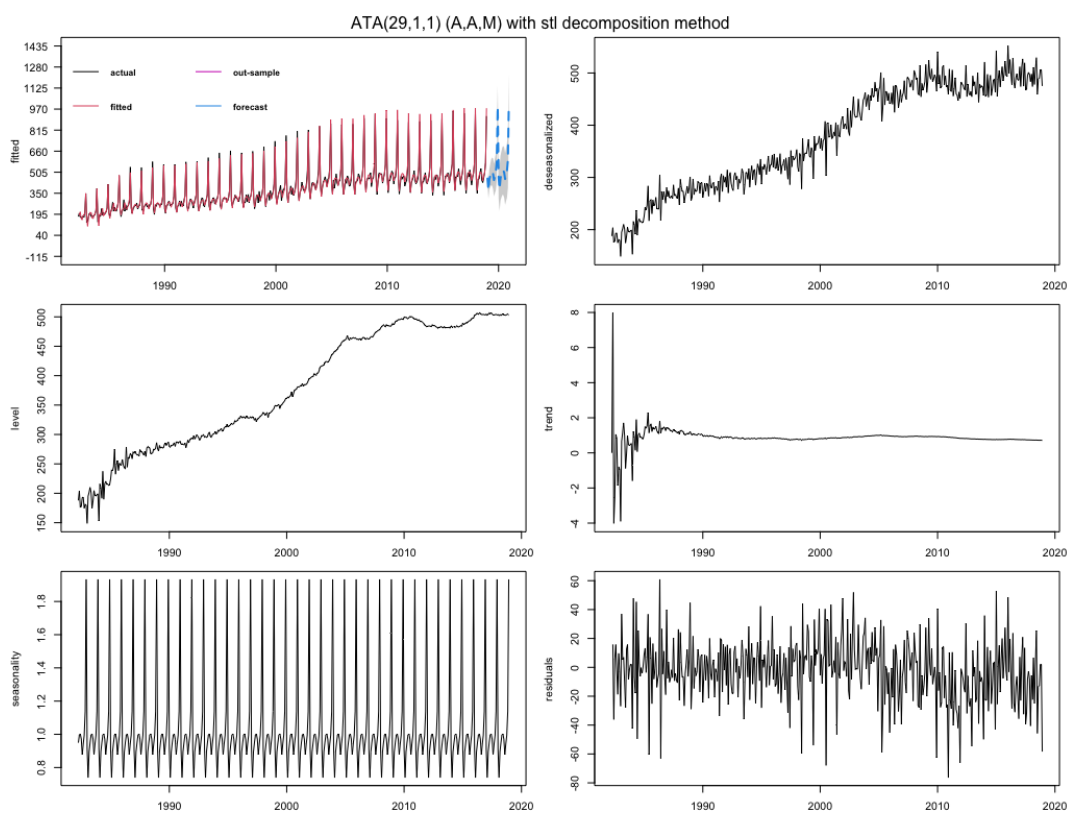

Figure 5: The Ata method with STL decomposition. 
- STL+ Decomposition (Hafen, 2010): The STL+ is implemented in stlplus R package. See more details in Hafen (2010).

fit_stlplus <- ATA(train_data, model.type = "A", seasonal.type = "M"

, seasonal.test $=$ TRUE, seasonal .model $=$ "stlplus", negative. forecast $=$ FALSE)
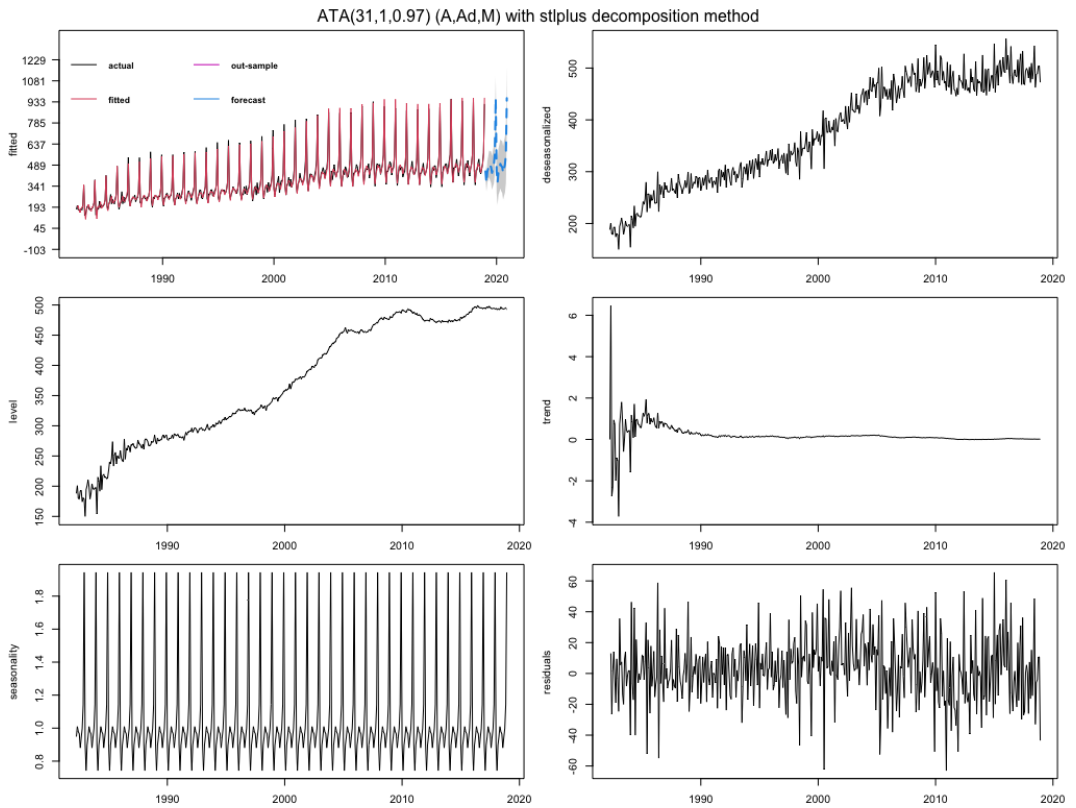

Figure 6: The Ata method with STLplus decomposition.

- TBATS Decomposition (Livera et al., 2011):TBATS uses Box-Cox transformation, exponential smoothing, trigonometric seasonality and ARMA errors (Livera et al., 2011).

fit_tbats <- ATA(train_data, seasonal.test = TRUE, seasonal.model = "tbats" , level.fixed $=$ TRUE, negative.forecast $=$ FALSE, plot. out $=$ TRUE)
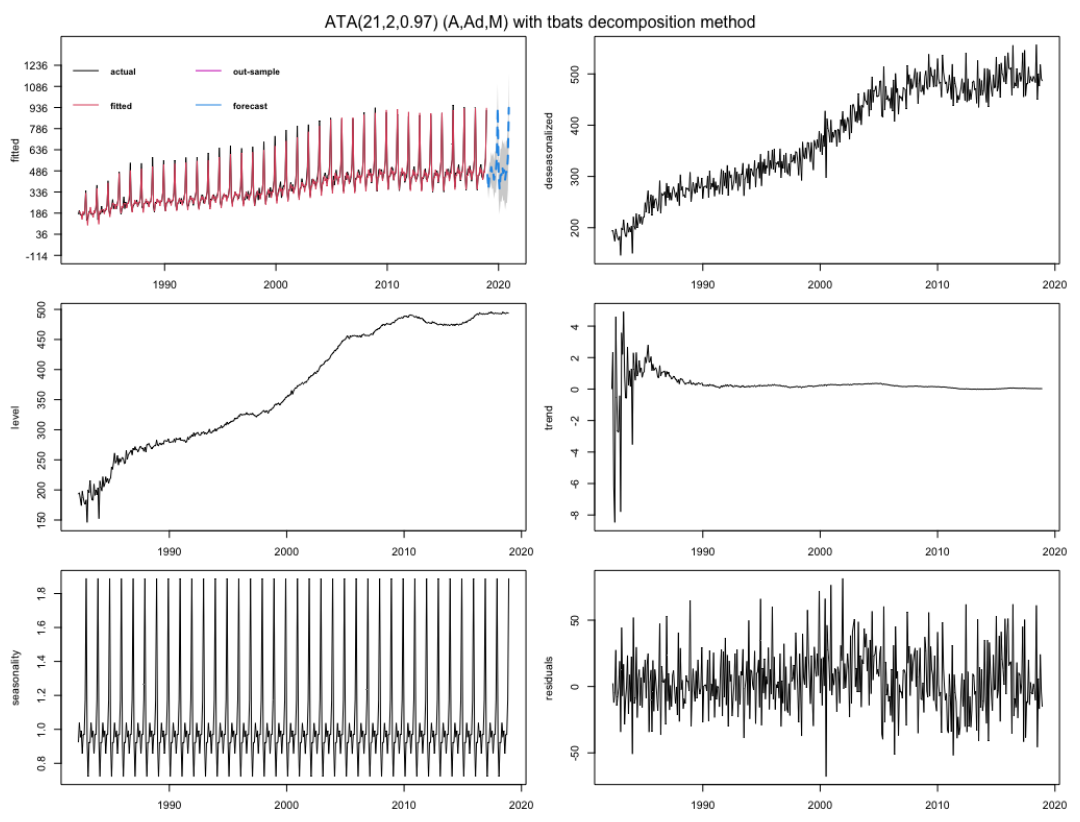

Figure 7: The Ata method with TBATS decomposition. 
- stR Decomposition (Dokumentov and Hyndman, 2015): Seasonal-Trend decomposition procedure based on Regression (stR) is similar to Ridge Regression, and Robust stR can be related to LASSO. The stR procedure grants for multiple seasonal and cyclic components and multiple linear regressors with constant, flexible, seasonal, and cyclic effect. The Seasonal-Trend decomposition by Regression is implemented in stR $\mathrm{R}$ package.

fit_stR <- ATA(train_data, seasonal.test = TRUE, seasonal.model = "stR" , negative. forecast $=$ FALSE, plot. out $=$ TRUE)
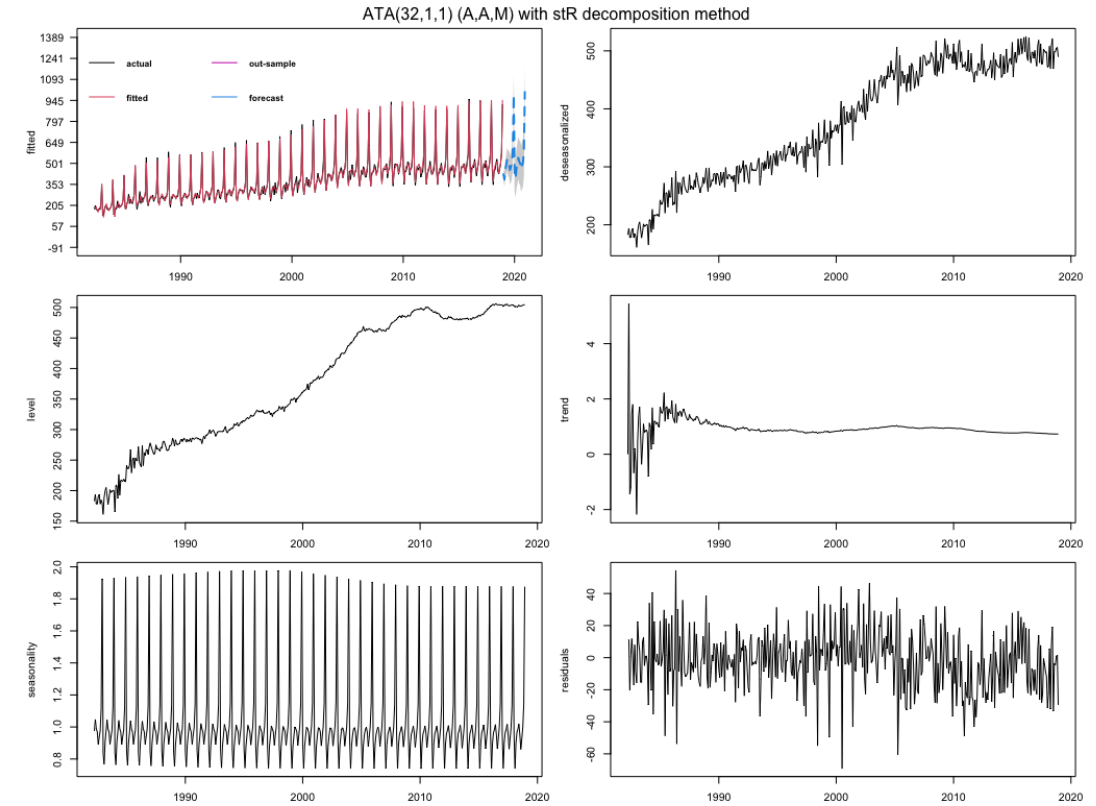

Figure 8: The Ata method with stR decomposition.

\section{Univariate time series forecasting with the Ata method}

Ata method is an innovative new forecasting technique where the forms of the models are similar to ES models. Still, the smoothing parameters depend on the sample size, are optimized on a discrete space. Initialization is both easier as it is done simultaneously when the parameters are optimized and is less influential since the weights assigned to initial values approach zero quickly. The Ata method can easily be applied to all time series settings and provides better forecasting performance due to its flexibility. ATA-damped is a version of the Ata method that mainly focuses on the trend component, allowing it to range both in magnitude and form.

For a time series $\left\{y_{1}, \ldots, y_{n}\right\}$, the Ata method can be given in additive form as below:

$$
\begin{aligned}
l_{t} & =\left(\frac{p}{t}\right) y_{t}+\left(\frac{t-p}{t}\right)\left(l_{t-1}+\phi b_{t-1}\right), \\
b_{t} & =\left(\frac{q}{t}\right)\left(l_{t}-l_{t-1}\right)+\left(\frac{t-q}{t}\right)\left(\phi b_{t-1}\right),
\end{aligned}
$$

where $p$ is the smoothing parameter for level, $q$ is the smoothing parameter for trend, $\phi$ is the dampening parameter and $l_{t}=y_{t}$ for $t \leq p, b_{t}=y_{t}-y_{t-1}$ for $t \leq q, b_{1}=0, p \in\{1,2, \ldots, n\}$, $q \in\{0,1,2, \ldots, p\}, \phi \in(0,1]$, and $p \geq q$. Then, the $h$ step ahead forecasts can be obtained by:

$$
\hat{y}_{t+h \mid t}=l_{t}+\left(\phi+\phi^{2}+\ldots+\phi^{h}\right) b_{t} .
$$

Similarly for a time series $\left\{y_{1}, \ldots, y_{n}\right\}$, the Ata method can be given in multiplicative form as below:

$$
l_{t}=\left(\frac{p}{t}\right) y_{t}+\left(\frac{t-p}{t}\right)\left(l_{t-1} b_{t-1}^{\phi}\right)
$$




$$
b_{t}=\left(\frac{q}{t}\right)\left(\frac{l_{t}}{l_{t-1}}\right)+\left(\frac{t-q}{t}\right)\left(b_{t-1}^{\phi}\right)
$$

where, again, $p$ is the smoothing parameter for level, $q$ is the smoothing parameter for trend, $\phi$ is the dampening parameter and $l_{t}=y_{t}$ for $t \leq p, b_{t}=\frac{y_{t}}{y_{t-1}}$ for $t \leq q, b_{1}=1, p \in\{1,2, \ldots, n\}$, $q \in\{0,1,2, \ldots, p\}, \phi \in(0,1]$, and $p \geq q$. Then, the $h$ step ahead forecasts can be obtained by:

$$
\hat{y}_{t+h \mid t}=l_{t}+b_{t}^{\left(\phi+\phi^{2}+\ldots+\phi^{h}\right)}
$$

Since both versions of the method require three parameters, we will distinguish between them by using the notation $A T A_{a d d}(p, q, \phi)$ for the additive form and $A T A_{\text {mult }}(p, q, \phi)$ for the multiplicative form.

Notice that when $q=0$, both forms of ATA are reduced to the simple form $A T A(p, 0, \phi)$ which can be written as:

$$
l_{t}=\left(\frac{p}{t}\right) y_{t}+\left(\frac{t-p}{t}\right) l_{t-1}
$$

where $p \in\{1,2, \ldots, n\}$ and $l_{t}=y_{t}$ for $t \leq p$. Forecasts then can be obtained by $\hat{y}_{t+h \mid t}=l_{t}$.

When $q \neq 0$ and $\phi=1$, the additive and multiplicative forms of ATA are reduced to the trended versions $A T A_{\text {add }}(p, q, 1)$ and $A T A_{\text {mult }}(p, q, 1)$, which are given below, respectively:

$$
\begin{gathered}
l_{t}=\left(\frac{p}{t}\right) y_{t}+\left(\frac{t-p}{t}\right)\left(l_{t-1}+b_{t-1}\right), \\
b_{t}=\left(\frac{q}{t}\right)\left(l_{t}-l_{t-1}\right)+\left(\frac{t-q}{t}\right)\left(b_{t-1}\right), \\
\hat{y}_{t+h \mid t}=l_{t}+h b_{t}
\end{gathered}
$$

and

$$
\begin{gathered}
l_{t}=\left(\frac{p}{t}\right) y_{t}+\left(\frac{t-p}{t}\right)\left(l_{t-1} b_{t-1}\right), \\
b_{t}=\left(\frac{q}{t}\right)\left(\frac{l_{t}}{l_{t-1}}\right)+\left(\frac{t-q}{t}\right)\left(b_{t-1}\right), \\
\hat{y}_{t+h \mid t}=l_{t}+b_{t}^{h} .
\end{gathered}
$$

To sum up, ATA can be given in 7 forms, namely the additive damped form $\operatorname{ATA}_{\text {add }}(p, q, \phi)$ (equations (1-3)), multiplicative damped form $A T A_{\text {mult }}(p, q, \phi)$ (equations (4-6)), simple form $A T A(p, 0, \phi)$ (equation (7)), additive trend form $A T A_{a d d}(p, q, 1)$ (equations (8-10)), and finally multiplicative trend form $A T A_{\text {mult }}(p, q, 1)$ (equations (11-13)).

Another distinction can be made based on the parameter optimization process used for these forms. Unless otherwise stated, the parameter values that minimized the in-sample one step ahead using selected accuracy measures such as SMAPE, MASE, or OWA are used as optimum values, and optimization is carried out for all the parameters simultaneously. However, in some cases, we realized that fixing the smoothing parameter for the level and then optimizing the trend parameter can be beneficial. We call these the "level-fixed" versions of ATA. The optimization is carried out for these models as follows:

1. Find the value of $p$ that minimized the in-sample one step ahead $s M A P E$ for $q=0$ and $\phi=1$. Call this value $p^{*}$.

2. Holding $p=p^{*}$ fixed optimize $q$ (and $\phi$ if needed), again, by minimizing the in-sample one step ahead $S M A P E$.

Models where the parameter optimization is carried out using the algorithm in 1. and 2. will receive the superscript (lf) an abbreviation for "level-fixed" such as $A T A_{\text {add }}^{l f}(p, q, \phi)$ or $A T A_{\text {mult }}^{l f}(p, q, \phi)$.

\section{Obtaining prediction interval}

For forecasting horizon $h$, the prediction interval is obtained by: 


$$
y_{n+h \mid n} \pm C_{h}
$$

where $C_{h}=\sqrt{h} Z_{\alpha / 2} S_{e}, Z_{\alpha / 2}$ is the Normal deviate corresponding to $(1-\alpha) \%$ confidence interval, and $S_{e}$ is the standard deviation of the one step ahead errors of model fitting. If any lower bounds are found to be negative, they are set equal to zero.

\section{Model selection}

There are plenty of measures and criteria available in the forecasting literature for interpreting the achievements and accuracy of forecasting methods. In the M-Competitions, some of these measures were employed without any obvious consensus as to the pros and cons of each.

A forecast error is a difference between an observed value and its forecast. Forecast errors are different from residuals in two aspects. Firstly, residuals are computed on the training set, while forecast errors are computed on the test set. Secondly, residuals are based on one-step-ahead forecasts, while forecast errors can contain multi-step forecasts (Hyndman and Athanasopoulos, 2019).

Let $Y_{t}$ indicates the observation at time $t$, and $F_{t}$ indicates the forecast of $Y_{t}$. The forecast error $e_{t}=Y_{t}-F_{t}$ is calculated. The forecasts are calculated from a common base time and are of varying forecast horizons. Hence, we calculate out of sample forecasts $F_{n+1}, \ldots, F_{n+m}$ based on data from times $t=1, \ldots, n$. Optionally, the forecasts can be from varying base times and be of a coherent forecast horizon. Namely, we can calculate forecasts $F_{1+h}, \ldots, F_{m+h}$ where each $F_{j+h}$ is based on data from times $t=1, \ldots, n$. The in-sample forecasts in the examples above were based on the second scenario with $h=1$. A third scenario shows up when we request to compare the accuracy of methods across many series at a forecast horizon. Then we calculate a single $F_{n+h}$ based on data from times $t=1, \ldots, n$ for each of $\mathrm{m}$ different series (Hyndman and Koehler, 2006). In this study, we adapt M4 and prior M-Competitions' accuracy measures pool.

\section{Automatic forecasting}

We unite the prior concepts to obtain a robust and widely appropriate automatic forecasting algorithm. The concept is summarized below.

1. Identify and correct for seasonality in time series, respectively.

- Detect stationarity and seasonality in time series.

- Decompose time series.

2. For the selected time series data, apply all models that are applicable, optimizing the parameters of the ATA model in each case.

3. Select the best of the ATA models according to the selected accuracy measure (SMAPE is default for the ATAforecasting package).

4. Generate point forecasts using the best model (with optimized parameters).

5. Obtain prediction intervals for the best model.

\section{ATAforecasting in practice}

This section introduces an overview of how the package is structured.

This software enables both numerical and graphical outputs to be displayed for all methods described in the previous section. This software is intended to be used with the R statistical program (R Core Team, 2016). Our package is composed of 13 functions that allow users to obtain estimates for all proposed methods. Details on the usage of the functions (described in Table 1) can be obtained with the corresponding help pages.

Returns ATA $(p, q, \phi)$ applied to $X$, based on the modified simple ES as described in Yapar (2018). The Ata method is a new univariate time series forecasting method that provides innovative solutions to issues faced during the initialization and optimization stages of existing methods. The ATA's forecasting performance is superior to existing methods both in terms of easy implementation and accurate forecasting. It can be applied to non-seasonal or deseasonalized time series, where the deseasonalization can be performed via any preferred decomposition method. This methodology performed extremely well on the M3 and M4-Competition data.

\section{Functions of ATAforecasting package}

Many functions, including ATA, ATA. Forecast, ATA. Plot, ATA. Print, ATA. Accuracy, ATA. Seasonality, ATA. Transform, ATA. BackTransform produce output in the form of a ATAforecasting object (i.e., an 
object of class "ata"). This package needs some R packages for unit root tests, seasonal unit root tests, seasonal decompositions, M3 dataset, M4 dataset, and benchmark forecast models to work consistently across a range of forecasting models. These R package are Rcpp (Eddelbuettel et al., 2020a), RcppArmadillo (Eddelbuettel et al., 2020b), tseries (Trapletti and Hornik, 2020), forecast (Hyndman et al., 2020), urca (Pfaff et al., 2016), uroot (de Lacalle, 2020), seasonal (Sax and Eddelbuettel, 2020), stR (Dokumentov and Hyndman, 2018), stlplus (Hafen, 2016), xts (Ryan et al., 2020), timeSeries (Wuertz et al., 2020), TSA (Chan and Ripley, 2020), Mcomp (Hyndman et al., 2018), M4Comp2018 (BenTaieb, 2018).

Objects of class "ata" contain information about the forecasting method, the data used, the point forecasts obtained, prediction intervals, residuals, and fitted values. There are several functions designed to work with these objects, including ATA. Forecast, ATA. Accuracy, ATA.Plot and ATA. Print.

\section{Description of the ATA function}

ATA function produces a ata object directly. If the first argument is of class ts (time series object) or msts (multi seasonal time series objects), it returns forecasts from the automatic ATA algorithm discussed in this chapter. The definition of ATA function is below.

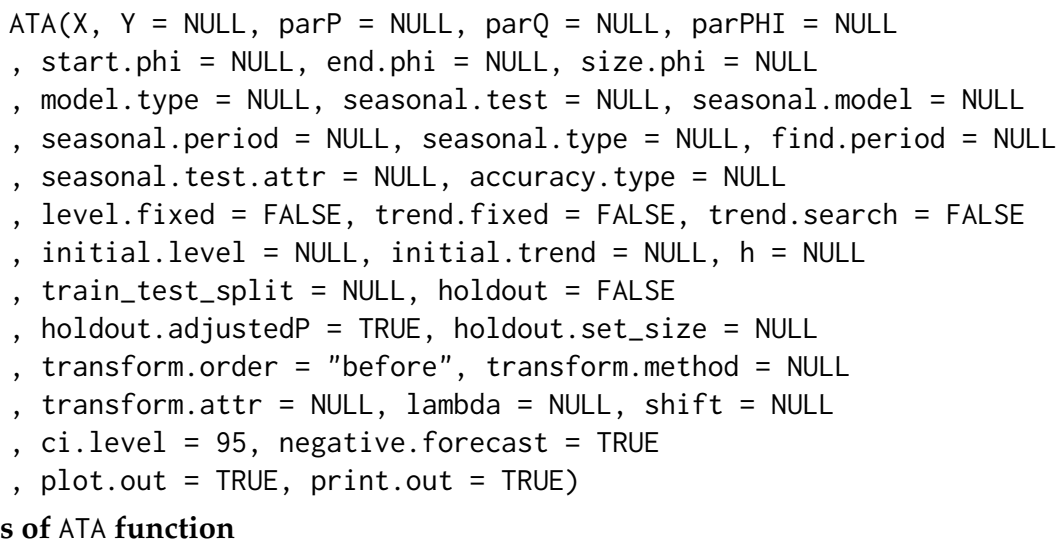

The ATA function works with many different types of inputs. It generally takes a time series data or time series model as its main argument, and produces forecasts appropriately. It always returns objects of class "ata".

If the first argument is of class ts or msts, it returns forecasts from the automatic ATA algorithm discussed in this chapter before.

- $X$ : A numeric vector or time series of class ts or msts for in-sample (trarining set).

- $Y$ : A numeric vector or time series of class ts or msts for out-sample (test set). If you do not have out-sample data, you can split in-sample data into training and test dataset with train_test_split argument.

- $h$ : The number of steps to forecast ahead. When the parameter is NULL; if the frequency of $X$ is 4 the parameter is set to 8 ; if the frequency of $X$ is 5 , the parameter is set to 10 ; if the frequency of $X$ is 12 , the parameter is set to 24 ; if the frequency of $X$ is 24 , the parameter is set to 48 ; the parameter is set to 6 for other cases.

- train_test_split : If $Y$ is NULL, this parameter divides $X$ into two parts: training set (in-sample) and test set (out-sample). train_test_split is number of periods for forecasting and size of test set. If the value is between 0 and 1, percentage of length is active.

- ci.level : Confidence Interval levels for forecasting.

- negative.forecast : Negative values are allowed for forecasting. Default value is TRUE. If FALSE, all negative values for forecasting are set to 0 .

- plot.out : Default is TRUE. If FALSE, graphics of Ata method are not shown.

- print.out : Default is TRUE. If FALSE, summary of Ata method is not shown.

\section{Level Parameters :}

- $\operatorname{parP}$ : Value of Level parameter $p$. If NULL or "opt", it is estimated. $p$ has all integer values from 1 to $\operatorname{length}(X)$.

- level.fixed : If TRUE, $p$ Star $Q$ is selected. First, fits $\operatorname{ATA}(p, 0)$ where $p=p^{*}$ is optimized for $q=0$. Then, fits $\operatorname{ATA}\left(p^{*}, q\right)$ where $q$ is optimized for $p=p^{*}$.

- initial.level : If NULL, FALSE is default. If FALSE, Ata method calculates the $p$ th observation in $X$ for level. If TRUE, Ata method calculates average of first $p$ value in $X$ for level. 


\section{Trend Parameters :}

- $\operatorname{par} Q$ : Value of Trend parameter $q$. If NULL or "opt", it is estimated. $q$ has all integer values from 0 to $p$.

- $\operatorname{parPHI}$ : Value of Damping Trend parameter $\phi$. If NULL or "opt", it is estimated. $\phi$ has all values from 0 to 1 .

- model.type : An one-character string identifying method using the framework terminology. The letter "A" for additive model, the letter "M" for multiplicative model. If NULL, both letters will be tried and the best model (according to the accuracy measure accuracy.type) returned.

- initial.trend: If NULL, FALSE is default. If FALSE, Ata method calculates the $q$ th observation in $X_{T}-X_{T-1}$ for trend. If TRUE, Ata method calculates average of first $q$ value in $X_{T}-X_{T-1}$ for trend.

- trend.opt :

1. none : none.

2. fixed: $p B$ ullet is selected. Fits $\operatorname{ATA}(\mathrm{p}, 1)$ where $\mathrm{p}=\mathrm{p}^{*}$ is optimized for $\mathrm{q}=1$.

3. search: $p$ Bullet is selected. Fits $\operatorname{ATA}(p, q)$ where $p=p^{*}$ is optimized for $q=q^{*}(q>0)$. Then, fits $\operatorname{ATA}\left(p^{*}, q\right)$ where $q$ is optimized for $p=p^{*}$.

- start.phi : Lower boundary for searching parPHI. If NULL, 0 is default.

- end.phi : Upper boundary for searching parPHI. If NULL, 1 is is default.

- size.phi : Increment step for searching parPHI. If NULL, the step size will be determined as the value that allows the bounds for the optimized value of parPHI to be divided into 20 equal parts.

Seasonal Parameters :

- seasonal.test : Testing for stationary and seasonality. If TRUE, the method firstly uses test = " $a d f$ ", Augmented Dickey-Fuller, unit-root test then the test returns the least number of differences required to pass the test at level $\alpha$. After the unit-root test, a seasonal test applies on the stationary $X$.

- seasonal.type: A one-character string identifying method for the seasonal component framework. If NULL, "M" is default. The letter "A" for additive model, the letter "M" for multiplicative model. If other seasonal decomposition method except decomp with "M", Box-Cox transformation with lambda $=0$ is selected.

- seasonal.model : A string identifying method for seasonal decomposition. If NULL, "decomp" method is default. c("none", "decomp", "stl", "stlplus", "tbats", "stR") phrases of methods denote.

- none : seasonal decomposition is not required.

- decomp : classical seasonal decomposition. If decomp, the stats package will be used.

- $s t l$ : seasonal-trend decomposition procedure based on LOESS developed by Cleveland et al. (1990). If stl, the stats package will be used.

- stlplus : seasonal-trend decomposition procedure based on LOESS developed by Cleveland et al. (1990). If stlplus, the stlplus package will be used.

- tbats : exponential smoothing state space model with Box-Cox transformation, ARMA errors, trend, and seasonal components as described in Livera et al. (2011). Parallel processing is used by default to speed up the computations. If tbats, the forecast package will be used.

- $s t R$ : seasonal-trend decomposition procedure based on regression developed by Dokumentov and Hyndman (2015). If $s t R$, the stR package will be used.

- $x 13$ : seasonal-trend decomposition procedure based on X13ARIMA/SEATS. If $x \mathbf{1 3}$, the seasonal package will be used.

- $x \mathbf{1 1}$ : seasonal-trend decomposition procedure based on X11. If $x \mathbf{1 1}$, the seasonal package will be used.

- seasonal.period : Value(s) of seasonal periodicity. If NULL, frequency of X is default If seasonal.period is not integer, $X$ must be an msts time series object. $\mathrm{c}(\mathrm{s} 1, \mathrm{~s} 2, \mathrm{~s} 3, \ldots)$ for multiple period. If $X$ has multiple periodicity, "tbats" or "stR" seasonal model have to be selected.

- seasonal.test.attr : Attributes set for unit root, seasonal unit root test, seasonality tests and X13ARIMA/SEATS, and X11. If you want to change, please use ATA. SeasAttr function and its output.

- find.period: Find seasonal period(s) automatically. If NULL, 0 is default. When find.period, 
- 0 : none.

-1 : single period with find. freq.

- 2 : single period with forecast: : findfrequency.

- 3 : multiple period with find. freq \& stR.

- $4:$ multiple period with find. freq \& tbats.

Accuracy Parameters :

- accuracy.type : Accuracy measure for selection of the best model. IF NULL, sMAPE is default.

1. lik: maximum likelihood functions.

2. sigma : residual variance.

3. $M A E:$ mean absolute error.

4. MSE : mean square error.

5. AMSE : average MSE over first 'nmse' forecast horizons.

6. RMSE : root mean squared error.

7. $M P E$ : mean percentage error.

8. MAPE : mean absolute percentage error.

9. $S M A P E:$ symmetric mean absolute percentage error.

10. MASE : mean absolute scaled error.

11. OWA : overall weighted average of MASE and SMAPE.

12. $M d A E:$ median absolute error.

13. MdSE : median square error.

14. RMdSE : root median squared error.

15. MdPE : median percentage error.

16. MdAPE : median absolute percentage error.

17. sMdAPE : symmetric median absolute percentage error.

- nmse : If accuracy.type == "AMSE", "nmse" provides the number of steps for average multistep MSE (' $2<=$ nmse $<=30$ ').

\section{Transform Parameters :}

- transform.order : If "before", Box-Cox transformation family will be applied, and then seasonal decomposition techniques will be applied. If "after", seasonal decomposition techniques will be applied, and then the Box-Cox transformation family will be applied.

- transform.method: Transformation methods: Box_Cox, Sqrt, Reciprocal, Log, NegLog, Modulus, BickelDoksum, Manly, Dual, YeoJohnson, GPower, GLog are used. Suppose the transformation process needs a shift parameter, ATA. Transform will calculate required the shift parameter automatically. When all types of Box-Cox family power techniques (except sqrt, reciprocal) are specified, model.type and seasonal.type is set to "A".

- transform.attr : Attributes set for Box-Cox transformation. If NULL, bcMethod = "loglik", $\mathrm{bcLower}=0$, bcUpper $=1, \mathrm{bcBias} A d j=$ FALSE. If you want to change, please use ATA. BoxCoxAttr function and its output.

- lambda : Box-Cox power transformation family parameter. If NULL, data transformed before the model is estimated.

- shift : Box-Cox power transformation family shifting parameter. If NULL, data transformed before the model is estimated. When lambda is specified, model.type and seasonal.type is set to "A". 


\section{Holdout Parameters :}

- holdout : Default is FALSE. If TRUE, Ata method uses the holdout forecasting for accuracy measure to select the best model. In holdout forecasting, the last few data points are removed from the data series. The remaining historical data series is called in-sample data (training set), and the holdout data is called out-of-sample data (holdout set). If TRUE, holdout.set_size will used for holdout data.

- holdout.adjustedP : Default is TRUE. If TRUE, parP will be adjusted by the length of trainingvalidation sets, and in-sample set when the holdout forecasting is active.

- holdout.set_size : If holdout is TRUE, this parameter will be the same as $h$ for defining holdout set.

- holdin : Default is FALSE. If TRUE, Ata method uses the hold-in forecasting for accuracy measure to select the best model. In hold-in forecasting, the last h-length data points are used for accuracy measure.

\section{Output of ATA function}

Returns an object of class "ata", containing the generic access or functions ATA. Forecast, and ATA. Accuracy extracts the useful features of the value returned by "ata" and associated functions.

- actual : The original time series.

- fitted : Fitted values (one-step forecasts). The mean is of the fitted values is calculated over the ensemble.

- level : Estimated level values.

- trend: Estimated trend values.

- residuals : Original values minus fitted values.

- coefp : The weights attached to level observations.

- coefq : The weights attached to trend observations.

- $p$ : Optimum level parameter.

- $q$ : Optimum trend parameter.

- phi : Optimum damped trend parameter.

- model.type : Form of trend.

- $h$ : The number of steps to forecast ahead.

- forecast : Point forecasts as a time series.

- out.sample : Test sets as a time series.

- method: The name of the optimum forecasting method as a character string.

- initial.level: Selected initial level values for the time series forecasting method.

- initial.trend: Selected initial trend values for the time series forecasting method.

- trend.opt : A choice of optional trend and level optimized trended methods (none, trend.fixed, or trend.search).

- transform.method : Box-Cox power transformation family methods are Box_Cox, Sqrt, Reciprocal, Log, NegLog, Modulus, BickelDoksum, Manly, Dual, YeoJohnson, GPower, GLog.

- transform.order : Define how to apply the Box-Cox power transformation techniques before or after seasonal decomposition.

- lambda: The Box-Cox power transformation family parameter.

- shift : The Box-Cox power transformation family shifting parameter.

- accuracy.type : Accuracy measure that is chosen for model selection.

- nmse : The number of steps for average multi-step MSE.

- accuracy : In-and out-sample accuracy measures and its descriptive that are calculated for optimum model are given.

- par.specs : Parameter sets for Information Criteria.

- holdout : Holdout forecasting is TRUE or FALSE.

- holdout.training : Training set in holdout forecasting.

- holdout.validation: Validation set in holdout forecasting.

- holdout.forecast : Holdout forecast. 
- holdout.accuracy : Accuracy measure chosen for model selection in holdout forecasting.

- is.season: Indicates whether it contains seasonal pattern.

- seasonal.model: The name of the selected decomposition method.

- seasonal.type : Form of seasonality.

- seasonal.period: The number of seasonality periods (which defaults to frequency $(X)$ ).

- seasonal.index : Weights of seasonality.

- seasonal : Estimated seasonal values.

- seasonal.adjusted: Deseasonalized time series values.

- execution.time : The real and CPU time (in seconds) spent by the system executing that task, including the time spent executing run-time or system services on its behalf.

- calculation.time : How much real time (in seconds) the currently running R process has already taken.

Here are quick start examples using "aus_retail" dataset monthly retail turnover (in million AUD) in Australian states from April 1982 to December 2018 in the tsibbledata package.

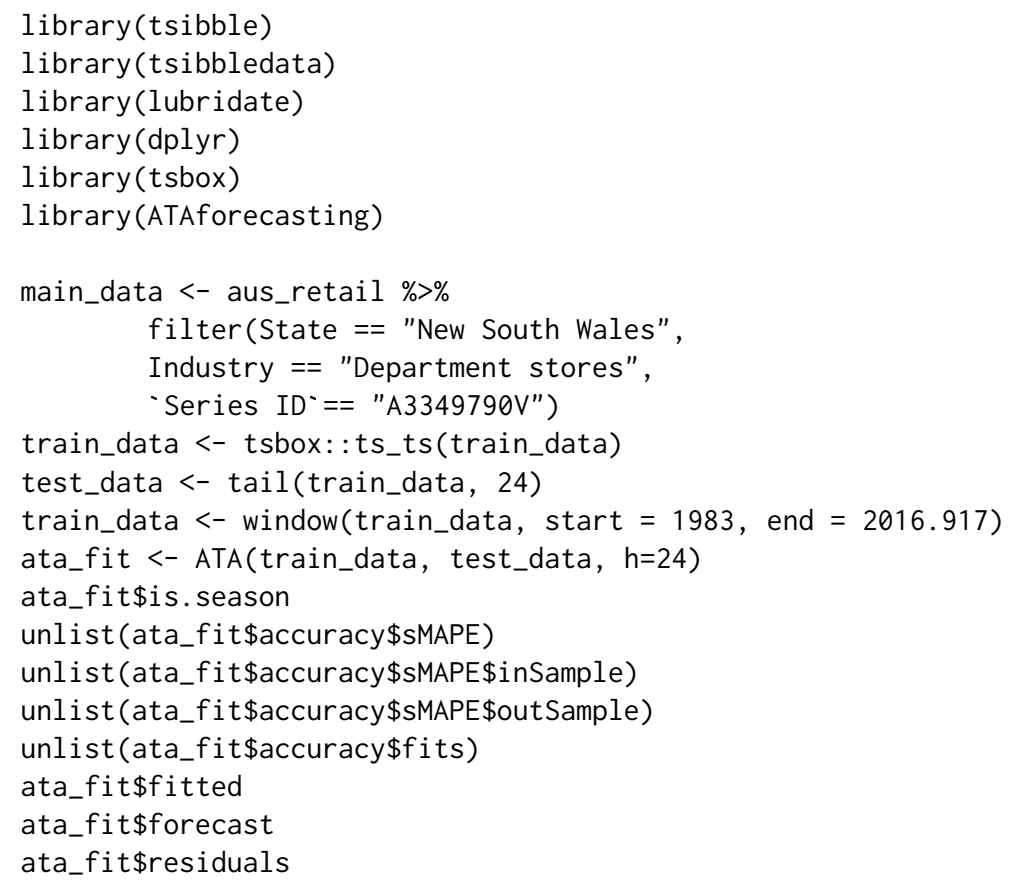

Here are some outputs for the above example from the ATAforecasting Package whose results are shown in Figure 9 and 10. 40 properties of the ATA module, including all results of the automatic forecasting using the Ata method are able to be obtained by using the "\$" command as shown in the above example. 

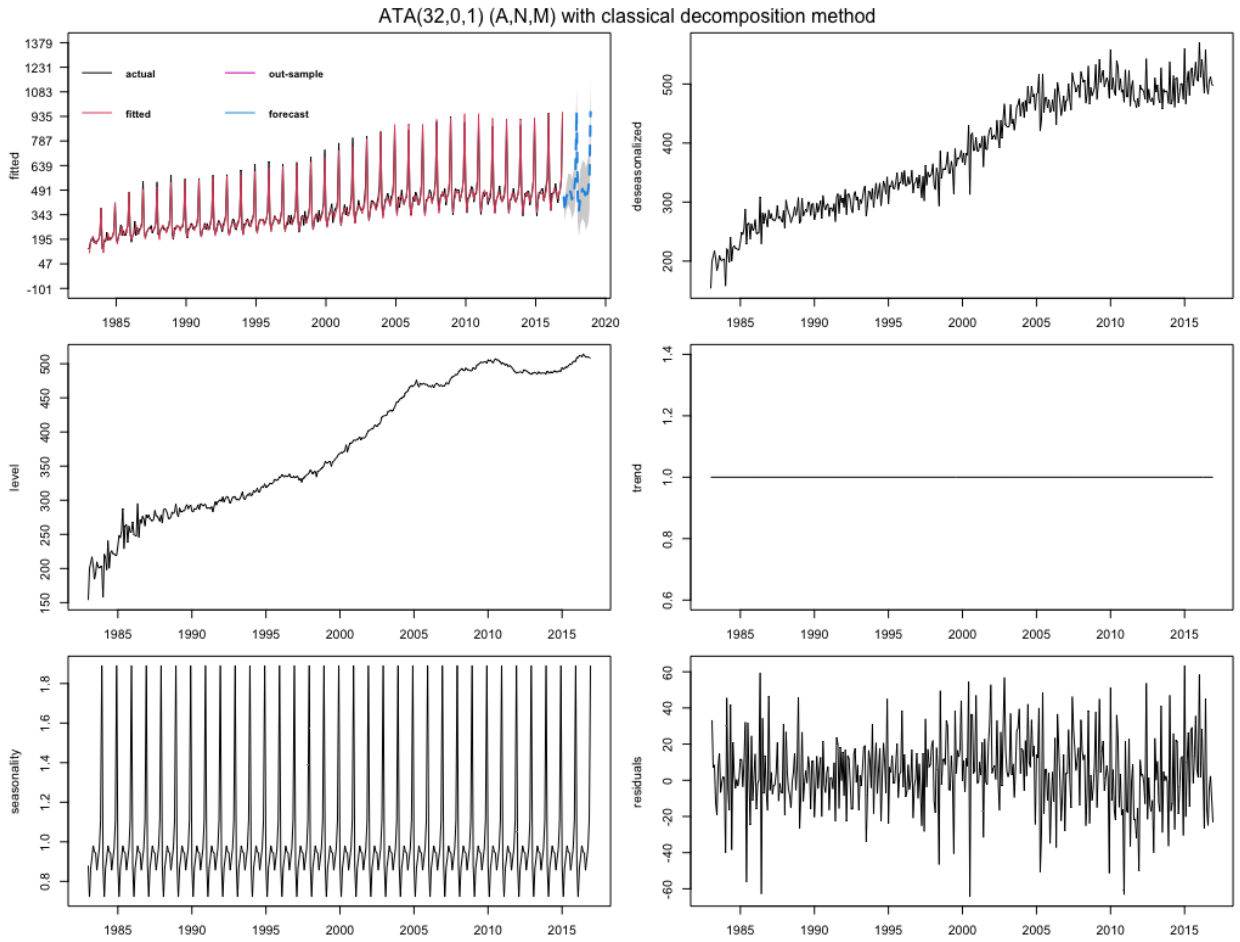

Figure 9: Forecasts from automatic ATA seasonal damped trended methods.

Another sample data is Makridakis Competitions 2000 (also known as the M-Competitions) monthly data in the Mcomp package (Hyndman et al., 2018).

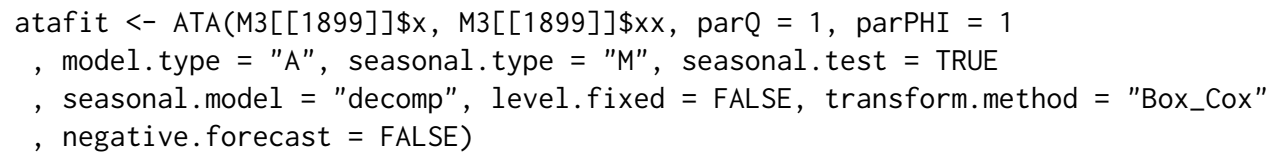

Here are some outputs for the above example from the ATAforecasting Package. The results are shown in Figure 11. 


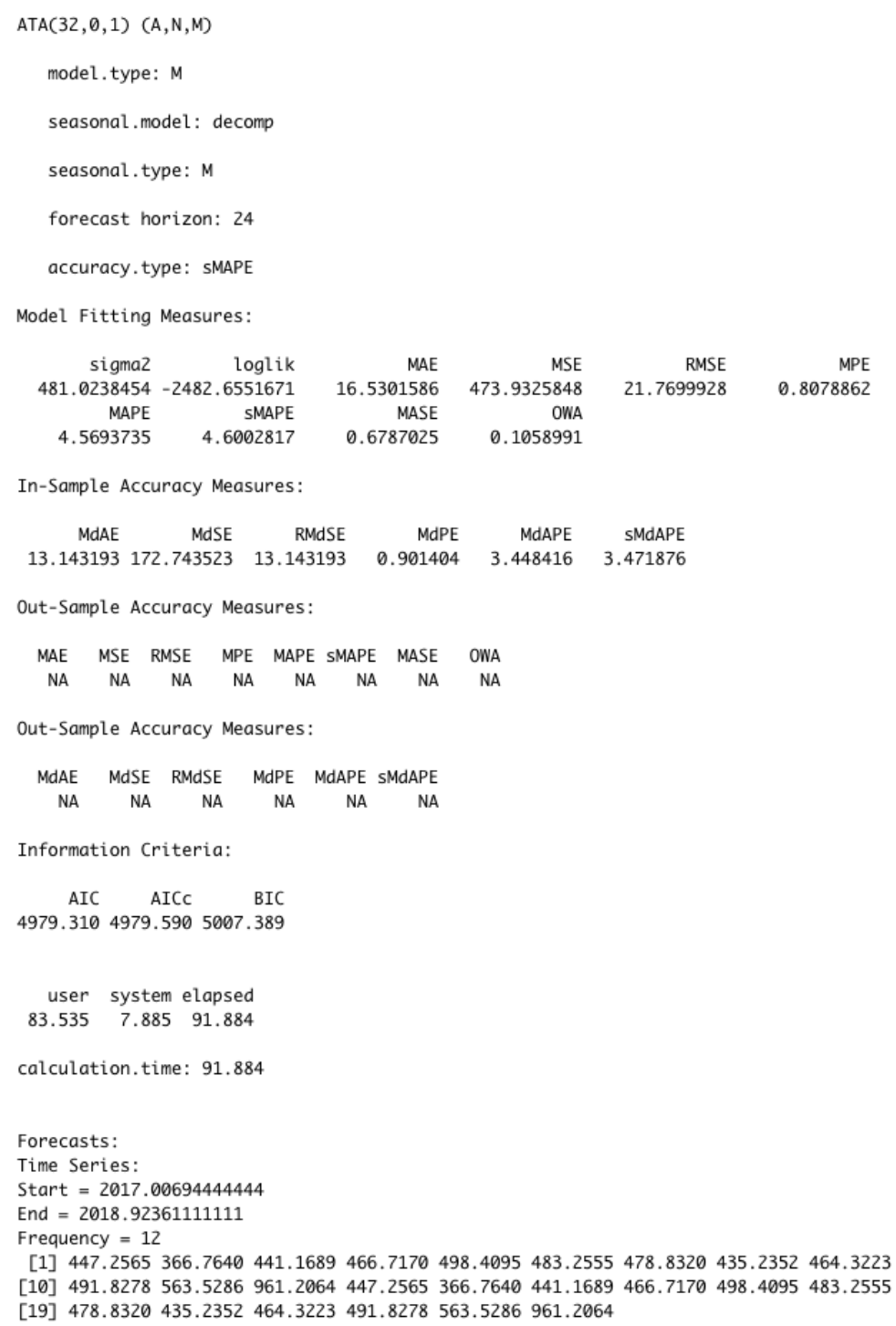

Figure 10: The default model output from the automatic ATA seasonal damped trended methods.

The object atafit is of class "ata" and contains all of the necessary information about the fitted model including model parameters, residuals, and so on. Printing the atafit object presents the main items of interest.

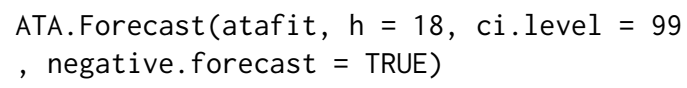

Some goodness-of-fit measures of forecast accuracy are obtained based on only the fitting data using ATA. Accuracy, we use the following commands.

$$
\text { ATA. Accuracy (atafit) }
$$

\section{Fable modeling wrappers for ATAforecasting}

We also developed a wrapper software (called fable.ata (Taylan et al., 2021b) to add the Ata method into the fable ecosystems using the fabletools ( $\mathrm{O}^{\prime} \mathrm{Hara}-W i l d$ et al., 2021b) package, which provides tools, helpers, and data structures for developing algorithms for the fable ecosystems ( $\mathrm{O}^{\prime}$ Hara-Wild et al., 2021a). Here are the quick start examples using the "aus_retail" dataset.

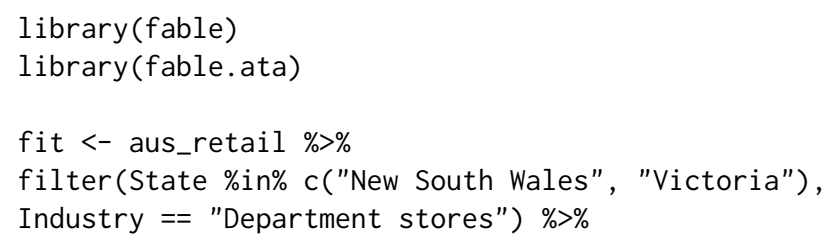



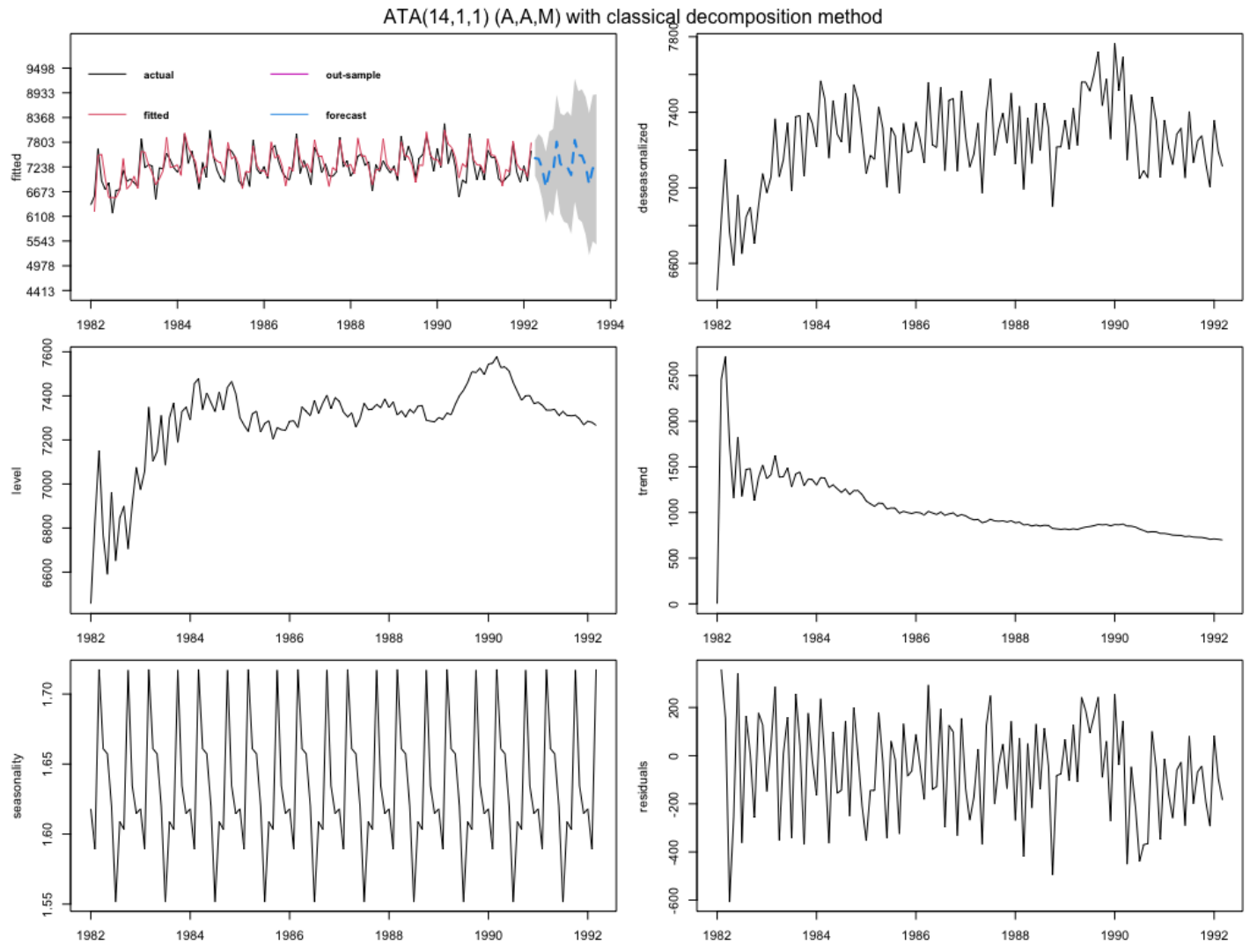

Figure 11: Forecasts from the automatic ATA seasonal trended methods for M3 sample.

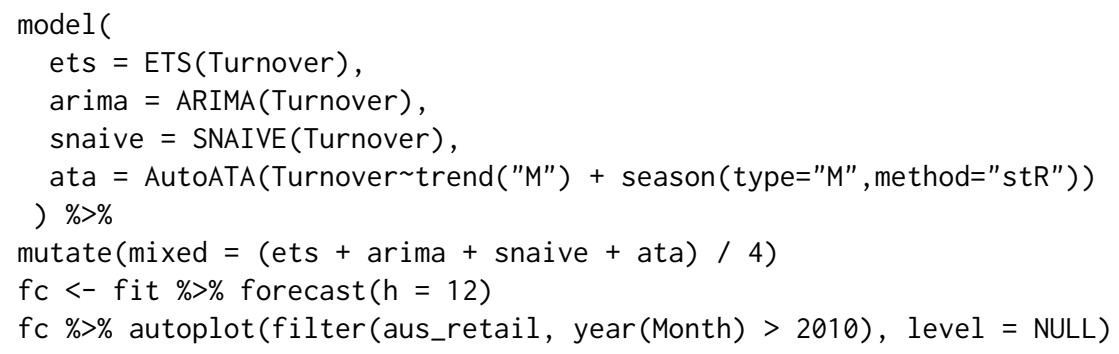

Here are some outputs for the above example from the fable ecosystem functions (fable and fable.ata packages). The results are shown in Figure 12 and Figure 13.

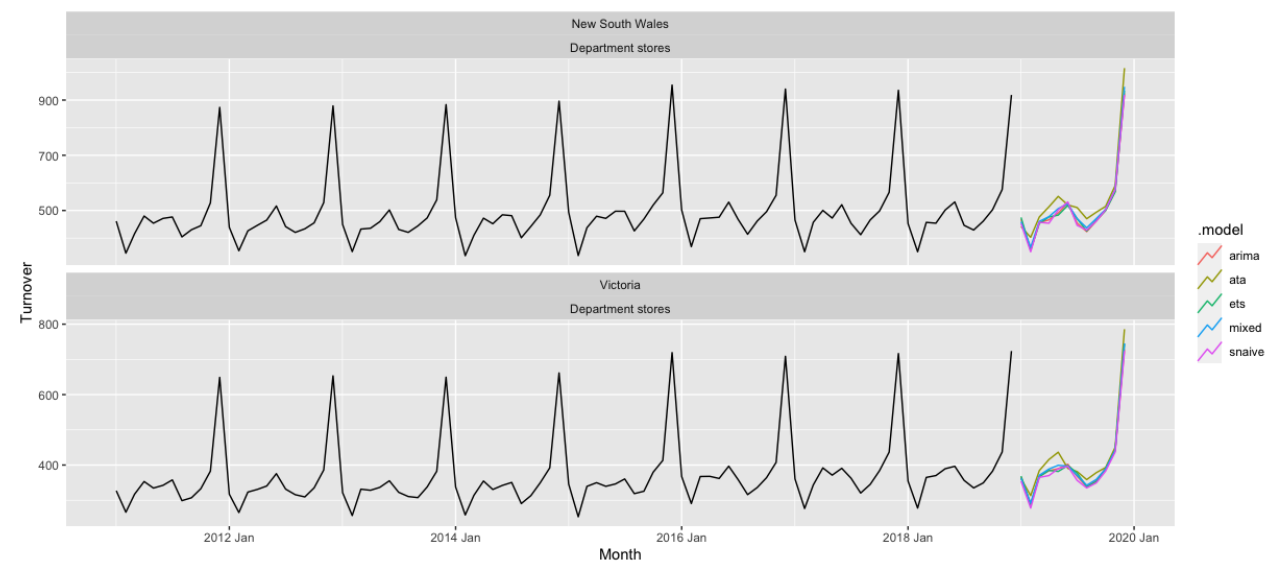

Figure 12: Forecasts from fable models for aus_retail dataset. 

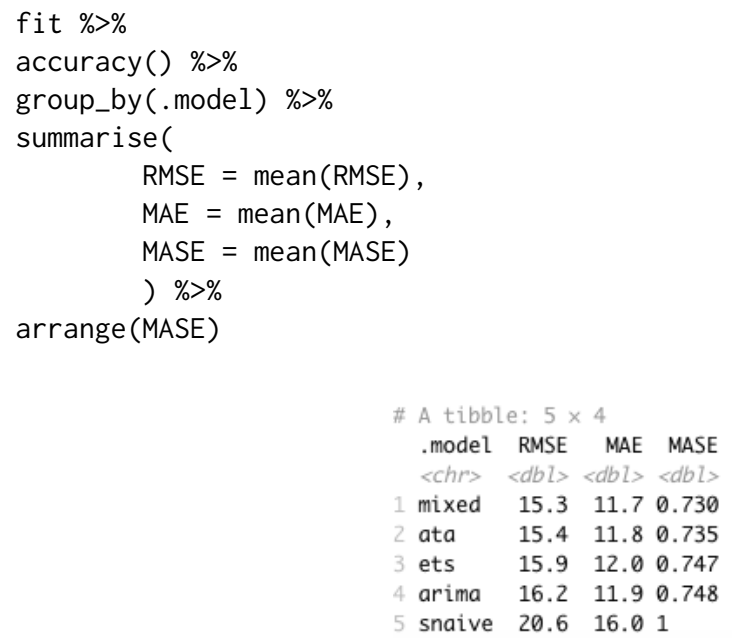

Figure 13: Comparison of fable models accuracy measures.

\section{Holdout forecasting}

Using holdout samples is substantial implementation to fit a model where the epoch of fit is dissimilar to the epoch of assessment. According to this model evaluation procedure, the epoch of fit completes at any moment before the last observation, and the rest of the data are held out as a non-overlapping epoch of assessment. In concern with the epoch of fit, the holdout sample is an epoch in the future, used to compare the forecasting accuracy of model fits to past data.

The concept of a holdout sample is to split the in-sample data into two parts. The last few data points are taken out from the in-sample data. The leftover data is called the training set, and the removed data is called the validation set or holdout set. Assume k periods have been taken out as holdout samples from a total of $\mathrm{T}$ periods. The parameters are optimized by minimizing the fit accuracy measure for the first part of the data. After the parameters are optimized, for each model, computed multi-step forecasts over the period covered by the second part, or holdout sample. The models are then evaluated, comparing accuracy measures for these out-of-sample multi-step predictions of the holdout sample. The model whose out-of-sample predictions best fit the holdout sample is chosen. The selected model is refitted using all the data to get the final forecasting model.

The ATAforecasting package makes it easy to use the holdout sample method of model selection. The time range used to fit models and the time range used for model evaluation are able to independently controlled. To use holdout samples, the period of evaluation range to that last part of the data, and the period of fit range to the remainder of the data are set. The automatic model selection feature is able to be used to select the model whose multiperiod out-of-sample predictions best fit the holdout sample.

Now, a quick start example of how to call the holdout method in the package.

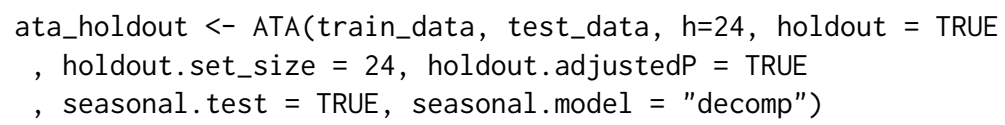

\section{Applications}

Ata method was proposed as an alternative to ES, and it is not a special case of it. The details on the method and how it helps solve some issues that ES suffers from can be found in Yapar et al. (2019), Yapar (2018), Yapar et al. (2018), Yapar et al. (2017). ATA can be adapted to all types of time series data and will always outperform its counter ES models.

There are many studies on the numerical and theoretical comparison of Box-Jenkins and ES methods. Several empirical studies have been published in turn by Reid (1969), Newbold and Granger (1974), Makridaki and Hibon (1979), Makridakis et al. (1982), Makridakis et al. (1993), Makridakis and Hibon (2000), Makridakis et al. (2018).

Efforts for better forecasting and the competitions in which the outcomes of these efforts are tested and measured will never cease. Better forecasting is crucial to every science and business field. The most important platforms in which the performance of the studies for accurate forecasting is measured 
are the M-Competitions (Hyndman, 2020). The most recent of these competitions, M4, has ended (Makridakis et al., 2018). The aim of the M4-Competition, like the competitions held before it, was to learn how to improve the forecasting accuracy, and how such learning can be applied to advance the theory and practice of forecasting, and are there any new methods that could really make a difference.

M-Competitions are very important and prestigious platforms for forecasting researchers since they provide researchers and developers of new forecasting methods opportunities to test and prove themselves. Another benefit of these competitions is that they usually lead to both the destruction of many taboos known in the forecasting literature and the discovery of new methods that help increase forecasting accuracy. The M-Competition was established by Spyros G. Makridakis in 1982 in a paper that studied the post-sample accuracy of several time series forecasting methods (Makridakis et al., 1982). The number of series was increased to 1001, and the data were subdivided into various categories (micro, macro, industry, demography, finance, other). The participants tested the accuracy of 24 methods on 1001 series with various horizons which were six for yearly data, eight for quarterly data, and eighteen for monthly data. The competition's goal was to explore how different procedures differ from each other and how information can be ensured that forecasters can make convenient choices under various conditions (Makridakis et al., 1984).

In Makridakis and Hibon (2000), the M3-Competition reports the reasons for conducting the competition and summarizes its outcomes. In the M3-Competition, 3003 series, composed of 6 different types of series and 4 different time intervals between successive observations. The three prior competitions have played a very major role in the forecasting literature. Their results ensured a basis for future forecasting research. Consequently, Makridakis et al. initiated the fourth competition. As per the Makridakis' team, the goal of the M4-Competition is to further study the utility and accuracy of various forecasting methods. Thus, the categories and number of the series and the forecasting methods are increased.

The M4-Competition is the progression of three previous competitions that began more than 45 years ago, whose objective was to learn how to evolve forecasting accuracy and how such learning can be implemented to proceed with the theory and performance of forecasting.

The purpose of M4 was to replicate the consequences of the prior ones and expand them into three aspects:

1. Substantially enhanced the number of series,

2. Contained machine learning forecasting methods,

3. Interpret both point forecasts and prediction intervals.

The some substantial outcomes of the M4-Competition are:

1. 12 of the 17 most accurate methods were "combinations" of mostly statistical approaches.

2. "hybrid" approach was a significant finding that use both statistical and machine learning features.

In the M4-Competition, the number of data from the previous M3-Competition (Makridakis and Hibon, 2000) was increased from 3,000 to 100,000. There were numerous applications (248), but only 49 of the applicants were able to provide forecasts for the entire 100,000 series. With the addition of 10 benchmarks and 2 standard methods, 61 methods were considered (Makridakis et al., 2020). Only 17 out of 49 valid applications outperformed the benchmark set by the competition committee. Of these 17 successful methods, 12 are combinations of known statistical methods obtained by using different weighting techniques.

The M3-Competition data set consists of 645 yearly, 756 quarterly, 1428 monthly, and 174 other series. The M4-Competition data set consists of 23000 yearly, 24000 quarterly, 48000 monthly, 359 weekly, 4227 daily, and 414 hourly series. The original data sets, as well as the forecasts of the methods that participated in the competitions, are available in the R packages Mcomp (Hyndman et al., 2018) and M4comp2018 (BenTaieb, 2018).

In order to test and to apply this approach's forecasting performance on real data and compare it to the benchmarks and especially counter ES models, forecasts obtained from five versions of it given the shortcode numbers Model-M3 and Model-M4 are fitted to the M3 and M4 competitions.

Therefore, in this implementation, predetermined model parameters as defined in the following list items are used to obtain accurate forecasts. Results from seven different applications of the Ata method will be considered here. 
1. $\operatorname{ATA}(p, 0,1)$ is an alternative to SES method where $p$ is the optimum value for $q=0$ with fixed damped trend $(\phi=1)$, and is where a simple model selection of the two models in $A T A^{\text {add }}(p, 0,1)$ and $A T A^{\text {mult }}(p, 0,1)$ is carried out based on selected in-sample accuracy measure.

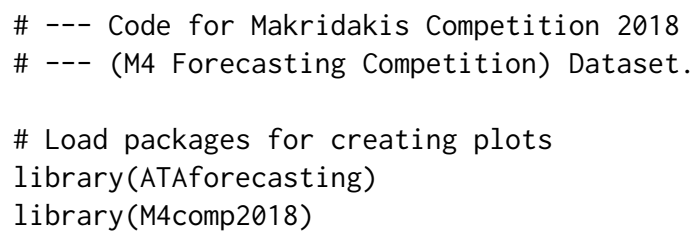

2. $A T A^{\text {add }}(p, 1,1)$ where $\mathrm{p}$ is optimized for $q=1$ with fixed damped trend $(\phi=1)$

fit2 <- ATA(M4[[1]]\$x, M4[[1]]\$xx, h = M4[[1]]\$h, parQ = 1

, parPHI $=1$, seasonal. test $=$ TRUE, seasonal. model $=$ "decomp"

, model.type = "A", accuracy. type = "SMAPE"

, negative.forecast $=$ FALSE)

3. ATA - comb where a simple average of the forecasts from the two models in (1) and (2) is used as a forecast.

fit3 $<-(f i t 1+f i t 2) / 2$

4. $A T A^{\text {add }}(p, 1, \phi)$ is an alternative to damped trend method where $q$ is optimized for $p=p^{*}$ with damped trend.

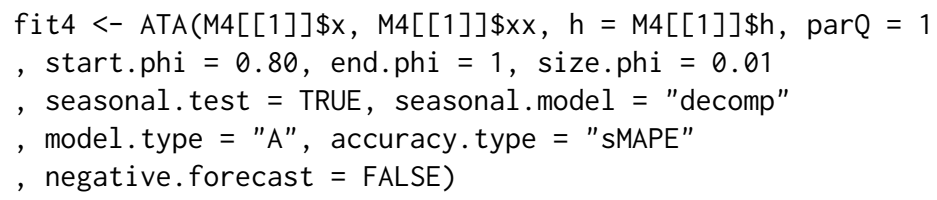

Model encoded by Model-M4 fits the $A T A^{a d d}(p, 1, \phi)$ to the yearly data sets and uses the ATA comb, a simple average of the forecasts obtained from the models $A T A^{\text {add }}(p, 1,1)$ and $A T A(p, 0,1)$, for the other data sets in M4-Competitions. Model-M3 fits $A T A^{\text {add }}(p, 0,1), A T A(p, 0,1)$, and uses the $A T A-c o m b$ for the data sets in M3-Competitions.

\begin{tabular}{|c|c|c|c|c|c|c|c|c|c|}
\hline Team & Method Type & Yearly & Quarterly & Monthly & Weekly & Daily & Hourly & Total & Rank \\
\hline Smyl & Hybrid & 13.176 & 9.679 & 12.126 & 7.817 & 3.170 & 9.328 & 11.374 & 1 \\
\hline Montero-Manso, et al. & Combination (S \& ML) & 13.528 & 9.733 & 12.639 & 7.625 & 3.097 & 11.506 & 11.720 & 3 \\
\hline Pawlikowski, et al. & Combination (S) & 13.943 & 9.796 & 12.747 & 6.919 & 2.452 & 9.611 & 11.845 & 5 \\
\hline Jaganathan. \& Prakash & Combination (S \& ML) & 13.712 & 9.809 & 12.487 & 6.814 & 3.037 & 9.934 & 11.695 & 2 \\
\hline Fiorucci \& Louzada & Combination (S) & 13.673 & 9.816 & 12.737 & 8.627 & 2.985 & 15.563 & 11.836 & 4 \\
\hline Petropoulos \& Svetunkov & Combination (S) & 13.669 & 9.800 & 12.888 & 6.726 & 2.995 & 13.167 & 11.887 & 6 \\
\hline Shaub & Combination (S) & 13.679 & 10.378 & 12.839 & 7.818 & 3.222 & 13.466 & 12.020 & 9 \\
\hline Legaki \& Koutsouri & Statistical & 13.366 & 10.155 & 13.002 & 9.148 & 3.041 & 17.567 & 11.986 & 8 \\
\hline Doornik, et al. & Combination (S) & 13.910 & 10.000 & 12.780 & 6.728 & 3.053 & 8.913 & 11.924 & 7 \\
\hline Pedregal, et al. & Combination (S) & 13.821 & 10.093 & 13.151 & 8.989 & 3.026 & 9.765 & 12.114 & 13 \\
\hline Model-M4 & Statistical & 13.930 & 10.292 & 12.936 & 8.540 & 3.095 & 12.851 & 12.098 & 11 \\
\hline Spiliotis \& Assimakopoulos & Statistical & 13.804 & 10.128 & 13.142 & 8.990 & 3.027 & 17.756 & 12.148 & 15 \\
\hline Roubinchtein & Combination (S) & 14.445 & 10.172 & 12.911 & 8.435 & 3.270 & 12.871 & 12.183 & 17 \\
\hline Ibrahim & Statistical & 13.677 & 10.089 & 13.321 & 9.089 & 3.071 & 18.093 & 12.198 & 18 \\
\hline Tartu M4 seminar & Combination (S \& ML) & 14.096 & 11.109 & 13.290 & 8.513 & 2.852 & 13.851 & 12.496 & 23 \\
\hline Waheeb & Combination (S) & 14.783 & 10.059 & 12.770 & 7.076 & 2.997 & 12.047 & 12.146 & 14 \\
\hline Darin \& Stellwagen & Statistical & 14.663 & 10.155 & 13.058 & 6.582 & 3.077 & 11.683 & 12.279 & 19 \\
\hline Dantas \& Cyrino Oliveira & Combination (S) & 14.746 & 10.254 & 13.462 & 8.873 & 3.245 & 16.941 & 12.553 & 25 \\
\hline The M4 Team (Theta) & Statistical & 14.593 & 10.311 & 13.002 & 9.093 & 3.053 & 18.138 & 12.309 & 20 \\
\hline The M4 Team (Com) & Statistical & 14.848 & 10.175 & 13.434 & 8.944 & 2.980 & 22.053 & 12.555 & 27 \\
\hline The M4 Team (Arima) & Statistical & 15.168 & 10.431 & 13.443 & 8.653 & 3.193 & 12.045 & 12.661 & 29 \\
\hline The M4 Team (Damped) & Statistical & 15.198 & 10.237 & 13.473 & 8.866 & 3.064 & 19.265 & 12.661 & 30 \\
\hline The M4 Team (ETS) & Statistical & 15.356 & 10.291 & 13.525 & 8.727 & 3.046 & 17.307 & 12.725 & 31 \\
\hline The M4 Team (Holt) & Statistical & 16.354 & 10.907 & 14.812 & 9.708 & 3.066 & 29.249 & 13.775 & 43 \\
\hline The M4 Team (SES) & Statistical & 16.396 & 10.600 & 13.618 & 9.012 & 3.045 & 18.094 & 13.087 & 37 \\
\hline
\end{tabular}

Table 2: Average forecasting errors for various data types and overall ranks with respect to sMAPE.

The forecasting performance of the Model-M4 that competed in the M4-Competition are given in the following three tables (Tables 2, 3, and 4) with respect to the error criteria SMAPE, MASE, and OWA, respectively. 
According to sMAPE (Table 2), the Model-M4 of the ATA models is ranked in the first 20. The Model-M4 performs much better than ETS despite the fact that only sMAPE was used for optimizing the ATA approaches for the in-sample data, and these approaches only considered limited numbers of candidate models to choose from, unlike ETS.

\begin{tabular}{|c|c|c|c|c|c|c|c|c|c|}
\hline Team & Method Type & Yearly & Quarterly & Monthly & Weekly & Daily & Hourly & Total & Rank \\
\hline Smyl & Hybrid & 2.980 & 1.118 & 0.884 & 2.356 & 3.446 & 0.893 & 1.536 & 1 \\
\hline Montero-Manso, et al. & Combination (S \& ML) & 3.060 & 1.111 & 0.893 & 2.108 & 3.344 & 0.819 & 1.551 & 3 \\
\hline Pawlikowski, et al. & Combination (S) & 3.130 & 1.125 & 0.905 & 2.158 & 2.642 & 0.873 & 1.547 & 2 \\
\hline Jaganathan. \& Prakash & Combination (S \& ML) & 3.126 & 1.135 & 0.895 & 2.350 & 3.258 & 0.976 & 1.571 & 6 \\
\hline Fiorucci \& Louzada & Combination (S) & 3.046 & 1.122 & 0.907 & 2.368 & 3.194 & 1.203 & 1.554 & 4 \\
\hline Petropoulos \& Svetunkov & Combination (S) & 3.082 & 1.118 & 0.913 & 2.133 & 3.229 & 1.458 & 1.565 & 5 \\
\hline Shaub & Combination (S) & 3.038 & 1.198 & 0.929 & 2.947 & 3.479 & 1.372 & 1.595 & 7 \\
\hline Legaki \& Koutsouri & Statistical & 3.009 & 1.198 & 0.966 & 2.601 & 3.254 & 2.557 & 1.601 & 8 \\
\hline Doornik, et al. & Combination (S) & 3.262 & 1.163 & 0.931 & 2.302 & 3.284 & 0.801 & 1.627 & 11 \\
\hline Pedregal, et al. & Combination (S) & 3.185 & 1.164 & 0.943 & 2.488 & 3.232 & 1.049 & 1.614 & 10 \\
\hline Model-M4 & Statistical & 3.117 & 1.231 & 0.962 & 2.578 & 3.277 & 2.238 & 1.631 & 13 \\
\hline Spiliotis \& Assimakopoulos & Statistical & 3.184 & 1.178 & 0.959 & 2.488 & 3.232 & 1.808 & 1.628 & 12 \\
\hline Roubinchtein & Combination (S) & 3.244 & 1.159 & 0.921 & 2.290 & 3.632 & 1.129 & 1.633 & 15 \\
\hline Ibrahim & Statistical & 3.075 & 1.185 & 0.977 & 2.583 & 3.894 & 2.388 & 1.644 & 16 \\
\hline Tartu M4 seminar & Combination (S \& ML) & 3.091 & 1.250 & 1.002 & 2.375 & 3.025 & 1.058 & 1.633 & 14 \\
\hline Waheeb & Combination (S) & 3.400 & 1.160 & 1.029 & 2.180 & 3.321 & 0.861 & 1.706 & 27 \\
\hline Darin \& Stellwagen & Statistical & 3.406 & 1.168 & 0.924 & 2.107 & 4.128 & 0.856 & 1.693 & 25 \\
\hline Dantas \& Cyrino Oliveira & Combination (S) & 3.294 & 1.170 & 0.952 & 2.534 & 3.436 & 1.598 & 1.657 & 17 \\
\hline The M4 Team (Theta) & Statistical & 3.382 & 1.232 & 0.970 & 2.637 & 3.262 & 2.455 & 1.696 & 26 \\
\hline The M4 Team (Com) & Statistical & 3.280 & 1.173 & 0.966 & 2.432 & 3.203 & 4.582 & 1.663 & 18 \\
\hline The M4 Team (Arima) & Statistical & 3.402 & 1.165 & 0.930 & 2.556 & 3.410 & 0.943 & 1.666 & 19 \\
\hline The M4 Team (Damped) & Statistical & 3.379 & 1.173 & 0.972 & 2.404 & 3.236 & 2.956 & 1.683 & 23 \\
\hline The M4 Team (ETS) & Statistical & 3.444 & 1.161 & 0.948 & 2.527 & 3.253 & 1.824 & 1.680 & 21 \\
\hline The M4 Team (Holt) & Statistical & 3.550 & 1.198 & 1.009 & 2.420 & 3.223 & 9.356 & 1.772 & 34 \\
\hline The M4 Team (SES) & Statistical & 3.981 & 1.340 & 1.019 & 2.685 & 3.281 & 2.385 & 1.885 & 39 \\
\hline
\end{tabular}

Table 3: Average forecasting errors for various data types and overall ranks with respect to MASE.

\begin{tabular}{|c|c|c|c|c|c|c|c|c|c|}
\hline Team & Method Type & Yearly & Quarterly & Monthly & Weekly & Daily & Hourly & Total & Rank \\
\hline Smyl & Hybrid & 0.778 & 0.847 & 0.836 & 0.851 & 1.046 & 0.440 & 0.821 & 1 \\
\hline Montero-Manso, et al. & Combination (S \& ML) & 0.799 & 0.847 & 0.858 & 0.796 & 1.019 & 0.484 & 0.838 & 2 \\
\hline Pawlikowski, et al. & Combination (S) & 0.820 & 0.855 & 0.867 & 0.766 & 0.806 & 0.444 & 0.841 & 3 \\
\hline Jaganathan \& Prakash & Combination (S \& ML) & 0.813 & 0.859 & 0.854 & 0.795 & 0.996 & 0.474 & 0.842 & 4 \\
\hline Fiorucci \& Louzada & Combination (S) & 0.802 & 0.855 & 0.868 & 0.897 & 0.977 & 0.674 & 0.843 & 5 \\
\hline Petropoulos \& Svetunkov & Combination (S) & 0.806 & 0.853 & 0.876 & 0.751 & 0.984 & 0.663 & 0.848 & 6 \\
\hline Shaub & Combination (S) & 0.801 & 0.908 & 0.882 & 0.957 & 1.060 & 0.653 & 0.860 & 7 \\
\hline Legaki \& Koutsouri & Statistical & 0.788 & 0.898 & 0.905 & 0.968 & 0.996 & 1.012 & 0.861 & 8 \\
\hline Doornik, et al. & Combination (S) & 0.836 & 0.878 & 0.881 & 0.782 & 1.002 & 0.410 & 0.865 & 9 \\
\hline Pedregal, et al. & Combination (S) & 0.824 & 0.883 & 0.899 & 0.939 & 0.990 & 0.485 & 0.869 & 11 \\
\hline Model-M4 & Statistical & 0.818 & 0.916 & 0.901 & 0.930 & 1.008 & 0.817 & 0.872 & 12 \\
\hline Spiliotis \& Assimakopoulos & Statistical & 0.823 & 0.889 & 0.907 & 0.939 & 0.990 & 0.860 & 0.874 & 13 \\
\hline Roubinchtein & Combination (S) & 0.850 & 0.885 & 0.881 & 0.873 & 1.091 & 0.586 & 0.876 & 14 \\
\hline Ibrahim & Statistical & 0.805 & 0.890 & 0.921 & 0.961 & 1.098 & 0.991 & 0.880 & 15 \\
\hline Tartu M4 seminar & Combination (S \& ML) & 0.820 & 0.960 & 0.932 & 0.892 & 0.930 & 0.598 & 0.888 & 17 \\
\hline Waheeb & Combination (S) & 0.880 & 0.880 & 0.927 & 0.779 & 0.999 & 0.507 & 0.894 & 18 \\
\hline Darin \& Stellwagen & Statistical & 0.877 & 0.887 & 0.887 & 0.739 & 1.135 & 0.496 & 0.895 & 19 \\
\hline Dantas \& Cyrino Oliveira & Combination (S) & 0.866 & 0.892 & 0.914 & 0.941 & 1.057 & 0.794 & 0.896 & 20 \\
\hline The M4 Team (Theta) & Statistical & 0.872 & 0.917 & 0.907 & 0.971 & 0.999 & 1.006 & 0.897 & 21 \\
\hline The M4 Team (Com) & Statistical & 0.867 & 0.890 & 0.920 & 0.926 & 0.978 & 1.556 & 0.898 & 22 \\
\hline The M4 Team (Arima) & Statistical & 0.892 & 0.898 & 0.903 & 0.932 & 1.044 & 0.524 & 0.902 & 23 \\
\hline The M4 Team (Damped) & Statistical & 0.890 & 0.893 & 0.924 & 0.917 & 0.997 & 1.141 & 0.907 & 25 \\
\hline The M4 Team (ETS) & Statistical & 0.903 & 0.891 & 0.915 & 0.931 & 0.996 & 0.852 & 0.908 & 26 \\
\hline The M4 Team (Holt) & Statistical & 0.947 & 0.932 & 0.988 & 0.966 & 0.995 & 2.749 & 0.971 & 37 \\
\hline The M4 Team (SES) & Statistical & 1.003 & 0.970 & 0.951 & 0.975 & 1.000 & 0.990 & 0.975 & 39 \\
\hline
\end{tabular}

Table 4: Average forecasting errors for various data types and overall ranks with respect to OWA.

The forecasting performance of the Model-M3 that competed in the M4-Competition are given in the Table 5 with the error criteria sMAPE.

These results should motivate users to consider ATA instead of ES-based forecasting. An important result from the M4-Competition was that combining forecasts improved accuracy. This improvement will become even stronger if the set of initial candidate models are chosen wisely and more meaningful if the combination can be obtained faster. Speed is an undeniable factor when choosing a forecasting method due to the need to obtain forecasts for the streaming and big data sets. The results obtained by using a simple combination of ARIMA and ATA for the M4-Competition data set are given in Table 6. For all error metrics considered, ATA approaches provide much better forecasts, and since the optimization is much faster than ETS, these more satisfying forecasts are obtained much faster.

Just by using the simple combination of ATA and ARIMA, forecasts that are more accurate than most of the methods that competed in the M4-Competition and that can compete with the more 


\begin{tabular}{|c|c|c|c|c|c|c|c|c|c|c|c|c|c|c|c|c|}
\hline \multirow[b]{2}{*}{ Method } & \multicolumn{10}{|c|}{ Forecasting horizons } & \multicolumn{5}{|c|}{ Averages } & \multirow[b]{2}{*}{$1-18$} \\
\hline & 1 & 2 & 3 & 4 & 5 & 6 & 8 & 12 & 15 & 18 & $1-4$ & $1-6$ & $1-8$ & $1-12$ & $1-15$ & \\
\hline Naive2 & 10.5 & 11.3 & 13.6 & 15.1 & 15.1 & 15.9 & 14.5 & 16.0 & 19.3 & 20.7 & 12.62 & 13.57 & 13.76 & 14.24 & 14.81 & 15.47 \\
\hline Single & 9.5 & 10.6 & 12.7 & 14.1 & 14.3 & 15.0 & 13.3 & 14.5 & 18.3 & 19.4 & 11.73 & 12.71 & 12.84 & 13.13 & 13.67 & 14.32 \\
\hline Holt & 9.0 & 10.4 & 12.8 & 14.5 & 15.1 & 15.8 & 13.9 & 14.8 & 18.8 & 20.2 & 11.67 & 12.93 & 13.11 & 13.42 & 13.95 & 14.60 \\
\hline Winter & 9.1 & 10.5 & 12.9 & 14.6 & 15.1 & 15.9 & 14.0 & 14.6 & 18.9 & 20.2 & 11.77 & 13.01 & 13.19 & 13.48 & 14.01 & 14.65 \\
\hline Dampen & 8.8 & 10.0 & 12.0 & 13.5 & 13.8 & 14.3 & 12.5 & 13.9 & 17.5 & 18.9 & 11.07 & 12.05 & 12.17 & 12.45 & 12.98 & 13.64 \\
\hline Comb (S-H-D) & 8.9 & 10.0 & 12.0 & 13.5 & 13.7 & 14.2 & 12.4 & 13.6 & 17.3 & 18.3 & 11.10 & 12.04 & 12.13 & 12.4 & 12.91 & 13.52 \\
\hline ETS & 8.8 & 9.8 & 12.0 & 13.5 & 13.9 & 14.7 & 13.0 & 14.1 & 17.6 & 18.9 & 11.04 & 12.13 & 12.32 & 12.66 & 13.14 & 13.77 \\
\hline$A T A(p, 0,1)$ & 8.9 & 10.0 & 12.1 & 13.7 & 13.9 & 14.7 & 12.8 & 13.9 & 17.3 & 18.9 & 11.16 & 12.21 & 12.34 & 12.64 & 13.13 & 13.77 \\
\hline$A T A(p, 1,1)$ & 8.4 & 9.7 & 11.5 & 12.9 & 13.6 & 14.2 & 12.9 & 15.4 & 18.9 & 20.9 & 10.64 & 11.72 & 11.94 & 12.66 & 13.32 & 14.09 \\
\hline $\operatorname{ATA}(p, q, \phi=0.5)$ & 8.6 & 9.6 & 11.6 & 13.2 & 13.5 & 14.2 & 12.4 & 13.7 & 17.0 & 18.6 & 10.76 & 11.77 & 11.92 & 12.24 & 12.75 & 13.39 \\
\hline Model-M3 & 8.5 & 9.6 & 11.4 & 12.8 & 13.0 & 13.6 & 12.0 & 13.1 & 16.3 & 17.4 & 10.56 & 11.47 & 11.58 & 11.94 & 12.40 & 12.94 \\
\hline
\end{tabular}

Table 5: Average sMAPE across different forecast horizons: all 3003 series.

\begin{tabular}{|c|c|c|c|c|c|c|c|}
\hline & Yearly & Quarterly & Monthly & Weekly & Daily & Hourly & Total \\
\hline & \multicolumn{7}{|c|}{ sMAPE } \\
\hline ETS \& ARIMA & 14.691 & 10.027 & 12.917 & 8.439 & 3.076 & 14.377 & 12.205 \\
\hline \multirow[t]{2}{*}{ Model-M3 \& ARIMA } & 13.847 & 9.987 & 12.653 & 7.607 & 2.998 & 11.942 & 11.859 \\
\hline & \multicolumn{7}{|c|}{ MASE } \\
\hline ETS \& ARIMA & 3.334 & 1.132 & 0.909 & 2.476 & 3.259 & 1.249 & 1.627 \\
\hline \multirow[t]{2}{*}{ Model-M3 \& ARIMA } & 3.093 & 1.148 & 0.908 & 2.345 & 3.255 & 1.436 & 1.575 \\
\hline & \multicolumn{7}{|c|}{ OWA } \\
\hline ETS \& ARIMA & 0.869 & 0.868 & 0.875 & 0.906 & 1.002 & 0.652 & 0.875 \\
\hline Model-M3 \& ARIMA & 0.813 & 0.872 & 0.866 & 0.837 & 0.989 & 0.625 & 0.849 \\
\hline
\end{tabular}

Table 6: Average forecasting errors for various data types and error metrics using simple combinations of forecasts.

accurate methods considering the computation complexity and time as important factors can be obtained. The results are given along with the ranks when all the methods are ranked according to OWA in Table 7. The three simple combinations of ATA and ARIMA are ranked in the top 10 when all other methods are considered.

\begin{tabular}{lllllllll}
\hline Team & Yearly & Quarterly & Monthly & Weekly & Daily & Hourly & Total & Rank \\
\hline Smyl & 0.778 & 0.847 & 0.836 & 0.851 & 1.046 & 0.440 & 0.821 & 1 \\
Montero-Manso, et al. & 0.799 & 0.847 & 0.858 & 0.796 & 1.019 & 0.484 & 0.838 & 2 \\
Pawlikowski, et al. & 0.820 & 0.855 & 0.867 & 0.766 & 0.806 & 0.444 & 0.841 & 3 \\
Jaganathan. \& Prakash & 0.813 & 0.859 & 0.854 & 0.795 & 0.996 & 0.474 & 0.842 & 4 \\
Fiorucci \& Louzada & 0.802 & 0.855 & 0.868 & 0.897 & 0.977 & 0.674 & 0.843 & 5 \\
Petropoulos \& Svetunkov & 0.806 & 0.853 & 0.876 & 0.751 & 0.984 & 0.663 & 0.848 & 6 \\
Model-M4 \& ARIMA & 0.813 & 0.872 & 0.866 & 0.837 & 0.989 & 0.625 & 0.849 & 8 \\
Shaub & 0.801 & 0.908 & 0.882 & 0.957 & 1.060 & 0.653 & 0.860 & 10 \\
Legaki \& Koutsouri & 0.788 & 0.898 & 0.905 & 0.968 & 0.996 & 1.012 & 0.861 & 11 \\
Doornik, et al. & 0.836 & 0.878 & 0.881 & 0.782 & 1.002 & 0.410 & 0.865 & 12 \\
\hline
\end{tabular}

Table 7: Average forecasting errors (OWA) for various data types along with the ranks.

\section{Conclusion}

In this study, we have introduced a novel method of bagging for the Ata method using power family transformations and various seasonal decomposition techniques. Ata method is a new and simple forecasting method that is an alternative to exponential smoothing. Although the Ata method's form is analogous to exponential smoothing, its weighting and parameterization schemes are utterly particular. Therefore, it is not a specific case of ES. It can be adapted to all types of time series data, much like ES and ARIMA, in addition to providing more accurate forecasts. Also, ATA can be optimized faster than exponential smoothing since its parameters can take on a limited number of discrete values only.

The goal of this manuscript is to introduce a new package for a new univariate time series forecasting method that provides innovative solutions to issues faced during the initialization and 
optimization stages of existing methods. The ATAforecasting package implements several different routines, most of which are related to the Ata method. Nevertheless, its modular structure enables the user to customize and complement the included functionality by means of custom algorithms or even other R packages. The ATAforecasting package provides a more general-purpose development as a comprehensive toolkit for automatic time series forecasting without any expertise on the R program. It focuses on modeling all types of time series components with any preferred Ata method and handling seasonality patterns by utilizing some popular decomposition techniques. Also, it combines several stationarity and seasonality tests, Box-Cox transformations, seasonal decomposition techniques with the Ata method. ATAforecasting performance is superior to existing methods both in terms of easy implementation, accurate, and flexible forecasting framework.

The ATAforecasting package categorizes some of the best-known techniques into three groups: (a) power transformation-based methods, (b) decomposition-based methods, and (c) time series forecasting-based methods. The package is also designed to assist research along with the whole modeling process: data preparation, model selection, prediction and forecasting, and interpretation of outcomes handling summaries and demonstrating functionalities. Providing these combinations of methods to the users is considered to introduce a new decomposition-based approach to time series forecasting with the Ata method, to provide automation, optimization, and bagging of the Ata method, which is an innovative and accurate univariate time series analysis method without any expertise by R program. Specifically, a proposed analytical methodology of the time series method with theATAforecasting $R$ package combines several stationarity and seasonality tests, power family transformations, and various seasonal decomposition techniques with the Ata method. In addition to this theoretical model, we focus on the computational implementation of all considered Ata methods in the ATAforecasting package. In particular, simulation and estimation have been demonstrated. Besides, the ATAforecasting package is aligned to many worthy R packages, such as forecast, urca, uroot, seasonal, stR, stlplus, xts, timeSeries, TSA, tseries.

In the future, the package should be extended to provide a comprehensive set of tools for three common issues in forecast combination prior to estimation, fast optimization of model parameters, missing values, and modeling with regressor variables. Users would have the option to automate the selection algorithm so that a good combination method is found based on the training set fit. Finally, the package offers specialized functions for summarizing and visualizing the combination results. Along this vein, a class for model specifications should be added alongside the actual implementations via arguments for the fitting functions. In that way, the package can be aligned to M-Competition benchmark time series models and useful R package. Furthermore, the package could benefit from robust estimation methods, another focus for future research.

\section{Acknowledgement}

This article was produced from Ali Sabri Taylan's PhD dissertation.

\section{Bibliography}

M. Adya, J. S. Armstrong, F. Collopy, and M. Kennedy. An application of rule-based forecasting to a situation lacking domain knowledge. International Journal of Forecasting, 16(4):477-484, 2000. [p508]

J. S. Armstrong. Combining forecasts: The end of the beginning or the beginning of the end? International Journal of Forecasting, 5(4):585-588, 1989. [p508]

J. S. Armstrong. Principles of forecasting: a handbook for researchers and practitioners, volume 30. Springer Science \& Business Media, 2001. [p508]

V. Assimakopoulos and K. Nikolopoulos. The theta model: a decomposition approach to forecasting. International Journal of Forecasting, 16(4):521-530, 2000. [p508]

J. M. Bates and C. W. Granger. The combination of forecasts. Journal of the Operational Research Society, 20(4):451-468, 1969. [p508]

S. BenTaieb. M4comp: Data from the M4 Time Series Forecasting Competition, 2018. URL https://github. com/cran/M4comp. R package version 0.0.1. [p523, 532]

C. Bergmeir, R. J. Hyndman, and J. M. Benítez. Bagging exponential smoothing methods using stl decomposition and box-cox transformation. International Journal of Forecasting, 32(2):303-312, 2016. [p507] 
P. J. Bickel and K. A. Doksum. An analysis of transformations revisited. Journal of the American Statistical Association, 76(374):296-311, 1982. [p511]

G. E. P. Box and D. R. Cox. An analysis of transformations. Journal of the Royal Statistical Society. Series B (Methodological), 26(2):211-252, 1964. [p510, 511]

R. G. Brown. Statistical forecasting for inventory control. McGraw/Hill, 1959. [p508]

J. P. Burman. Seasonal adjustment by signal extraction. Journal of the Royal Statistical Society. Series A (General), 143(3):321-337, 1980. [p515]

F. Canova and B. E. Hansen. Are seasonal patterns constant over time? a test for seasonal stability. Journal of Business and Economic Statistics, 13(3):237-252, 1995. [p514]

R. J. Carroll. A robust method for testing transformations to achieve approximate normality. Journal of the Royal Statistical Society. Series B (Methodological), 42(1):71-78, 1980. [p510]

B. Cetin and I. Yavuz. Comparison of forecast accuracy of Ata and exponential smoothing. Journal of Applied Statistics, 0(0):1-11, 2020. doi: 10.1080/02664763.2020.1803813. [p507]

K.-S. Chan and B. Ripley. TSA: Time Series Analysis, 2020. URL https://cran.r-project.org/ package=TSA. R package version 1.3. [p523]

G. Chen, R. A. Lockhart, and M. A. Stephens. Box-cox transformations in linear models: Large sample theory and tests of normality. Canadian Journal of Statistics, 30(2):177-209, 2002. [p510]

R. T. Clemen. Combining forecasts: A review and annotated bibliography. International Journal of Forecasting, 5(4):559-583, 1989. [p508]

R. B. Cleveland, W. S. Cleveland, J. E. McRae, and I. Terpenning. Stl: A seasonal-trend decomposition procedure based on loess. Journal of Official Statistics, 6(1):3-73, 1990. [p508, 515, 518, 524]

J. J. Commandeur, S. J. Koopman, and M. Ooms. Statistical software for state space methods. Journal of Statistical Software, 41(1), 2011. URL https://www. jstatsoft.org. [p515]

M. T. Copeland. Statistical indices of business conditions. The Quarterly Journal of Economics, 29(3): 522-562, 1915. [p515]

E. B. Dagum. X-11-ARIMA/88 Seasonal Adjustment Method - Foundations and Users' Manual. Statistics Canada, 1988. URL https://www. census.gov/ts/papers/Emanual.pdf. [p515]

E. B. Dagum and S. Bianconcini. Seasonal Adjustment Methods and Real Time Trend-Cycle Estimation. Springer, 2016. [p515]

R. Davidson and J. MacKinnon. Estimation and Inference in Econometrics. Oxford University Press, 1993. [p513]

J. L. de Lacalle. uroot: Unit Root Tests for Seasonal Time Series, 2020. URL https://cran. r-project. org/package=uroot. $\mathrm{R}$ package version 2.1-2. [p523]

D. A. Dickey and W. A. Fuller. Distribution of the estimators for autoregressive time series with a unit root. Journal of the American Statistical Association, 74(366):427-431, 1979. [p513]

D. A. Dickey, D. P. Hasza, and W. A. Fuller. Testing for unit roots in seasonal time series. Journal of the American Statistical Association, 79(386):355-367, 1984. [p514]

A. Dokumentov and R. J. Hyndman. Str: A seasonal-trend decomposition procedure based on regression. Monash Econometrics and Business Statistics Working Papers 13/15, Monash University, Department of Econometrics and Business Statistics, 2015. URL https: //EconPapers. repec. org/ RePEc:msh:ebswps:2015-13. [p515, 520, 524]

A. Dokumentov and R. J. Hyndman. stR: STR Decomposition, 2018. URL https://cran. r-project. org/package=stR. R package version 0.4. [p509,523]

B. P. Durbin, J. S. Hardin, D. M. Hawkins, and D. M. Rocke. A variance-stabilizing transformation for gene-expression microarray data. Bioinformatics, 18(1):105-110, 2002. [p511]

D. Eddelbuettel, R. Francois, J. J. Allaire, K. Ushey, Q. Kou, N. Russell, D. Bates, and J. Chambers. Rcpp: Seamless R and C++ Integration, 2020a. URL https://cran. r-project.org/package=Rcpp. R package version 1.0.5. [p523] 
D. Eddelbuettel, R. Francois, D. Bates, and B. Ni. RcppArmadillo: 'Rcpp' Integration for the 'Armadillo' Templated Linear Algebra Library, 2020b. URL https://cran. r-project.org/package=RcppArmadillo. $\mathrm{R}$ package version 0.10.1.2.0. [p523]

D. F. Findley. Some recent developments and directions in seasonal adjustment. Journal of official statistics, 21(2):343, 2005. [p515]

D. F. Findley, B. C. Monsell, W. R. Bell, M. C. Otto, and B.-C. Chen. New capabilities and methods of the x-12-arima seasonal-adjustment program. Journal of Business \& Economic Statistics, 16(2):127-152, 1998. [p515]

E. S. Gardner and E. McKenzie. Forecasting trends in time series. Management Science, 31(10):1237-1246, 1985. [p508]

V. M. Guerrero. Time-series analysis supported by power transformations. Journal of Forecasting, 12(1): 37-48, 1993. [p511]

R. Hafen. stlplus: Enhanced Seasonal Decomposition of Time Series by Loess, 2016. URL https://cran . rproject. org/package=stlplus. R package version 0.5.1. [p509, 523]

R. P. Hafen. Local regression models: Advancements, applications, and new methods. PhD thesis, Purdue University, 2010. [p519]

J. D. Hamilton. Time Series Analysis. Princeton University Press, 1994. [p513]

A. K. Han. A non-parametric analysis of transformations. Journal of Econometrics, 35(2-3):191-209, 1987. [p510]

A. C. Harvey. Forecasting, Structural Time Series Models and the Kalman Filter. Cambridge University Press, 1990. [p515]

F. Hayashi. Econometrics. Princeton University Press, 2000. [p513]

F. Hernandez and R. A. Johnson. The large-sample behavior of transformations to normality. Journal of the American Statistical Association, 75(372):855-861, 1980. [p510]

J. Horowitz. Semiparametric and Nonparametric Methods in Econometrics. Springer, 2009. [p510]

S. Hylleberg, R. F. Engle, C. W. J. Granger, and B. S. Yoo. Seasonal integration and cointegration. Journal of Econometrics, 1344(1):215-238, 1990. [p514]

R. J. Hyndman. A brief history of forecasting competitions. International Journal of Forecasting, 36(1): 7-14, 2020. [p532]

R. J. Hyndman and G. Athanasopoulos. Forecasting: principles and practice. OTexts, 2019. URL https://otexts.com/fpp3/. [p508,522]

R. J. Hyndman and A. B. Koehler. Another look at measures of forecast accuracy. International Journal of Forecasting, 22(4):679-688, 2006. [p522]

R. J. Hyndman, A. B. Koehler, R. D. Snyder, and S. Grose. A state space framework for automatic forecasting using exponential smoothing methods. International Journal of Forecasting, 18(3):439-454, 2002. [p508]

R. J. Hyndman, A. B. Koehler, J. K. Ord, and R. D. Snyder. Forecasting with exponential smoothing: the state space approach. Springer-Verlag, 2008. [p508]

R. J. Hyndman, M. Akram, C. Bergmeir, and M. O'Hara-Wild. Mcomp: Data from the M-Competitions, 2018. URL https://cran.r-project.org/package=Mcomp. R package version 2.8. [p523, 528, 532]

R. J. Hyndman, G. Athanasopoulos, C. Bergmeir, G. Caceres, L. Chhay, M. O'Hara-Wild, F. Petropoulos, S. Razbash, E. Wang, and F. Yasmeen. forecast: Forecasting functions for time series and linear models, 2020. URL https://cran. r-project. org/package=forecast. R package version 8.13. [p508, 509, 523]

J. A. John and N. R. Draper. An alternative family of transformations. Journal of the Royal Statistical Society Series C, 29(2):190-197, 1980. [p511]

D. M. Kelmansky, E. J. Martinez, and V. Leiva. A new variance stabilizing transformation for gene expression data analysis. Statistical Applications in Genetics and Molecular Biology, 12(6):653-666, 2013. [p511] 
A. J. Koning, P. H. Franses, M. Hibon, and H. O. Stekler. The m3 competition: Statistical tests of the results. International Journal of Forecasting, 21(3):397-409, 2005. [p508]

D. Kwiatkowski, P. Phillips, P. Schmidt, and Y. Shin. Testing the null hypothesis of stationarity against the alternative of a unit root: How sure are we that economic time series have a unit root? Journal of Econometrics, 54(1-3):159-178, 1992. [p513]

A. M. D. Livera, R. J. Hyndman, and R. D. Snyder. Forecasting time series with complex seasonal patterns using exponential smoothing. Journal of the American Statistical Association, 106(496):15131527, 2011. [p508, 515, 519, 524]

G. M. Ljung and G. E. P. Box. On a measure of lack of fit in time series models. Biometrika, 65(2): 297-303, 1978. [p514]

F. R. Macaulay. Appendix I - The Measurement of Probable Seasonal Fluctuations By Means of Operations on the Deviations of the Data, pages 121-136. NBER, 1931. URL http://www. nber . org/chapters/c9369. [p515]

S. G. Makridaki and M. Hibon. Accuracy of forecasting: an empirical investigation. Journal of Royal Statistical Society, 142(2):97-145, 1979. [p531]

S. Makridakis and R. L. Winkler. Averages of forecasts: Some empirical results. Management Science, 29 (9):987-996, 1983. [p508]

S. G. Makridakis and M. Hibon. The m3-competition: Results, conclusions and implications. International Journal of Forecasting, 16(4):451-476, 2000. [p508, 531, 532]

S. G. Makridakis, A. Andersen, R. Carbone, R. Fildes, M. Hibon, R. Lewandowski, J. Newton, E. Parzen, and R. Winkler. The accuracy of extrapolation (time series) methods: Results of a forecasting competition. Journal of Forecasting, 1(2):111-153, 1982. [p508, 531, 532]

S. G. Makridakis, A. Andersen, R. Carbone, R. Fildes, M. Hibon, R. Lewandowski, J. Newton, E. Parzen, and R. Winkler. The forecasting accuracy of major time series methods. Wiley, 1984. [p508, 532]

S. G. Makridakis, M. Hibon, S. Chatfield, M. Lawrence, T. Mills, K. Ord, and L. F. Simmons. The m-2 competition: a real time judgementally based forecasting study. International Journal of Forecasting, 9 (2):5-23, 1993. [p531]

S. G. Makridakis, E. Spiliotis, and V. Assimakopoulos. The $\mathrm{m} 4$ competition: Results, findings, conclusion and way forward. International Journal of Forecasting, 34(4):802-808, 2018. [p508, 531, 532]

S. G. Makridakis, E. Spiliotis, and V. Assimakopoulos. The m4 competition: 100,000 time series and 61 forecasting methods. International Journal of Forecasting, 36(1):54-74, 2020. [p532]

S. Meintanis and G. Stupfler. Transformations to symmetry based on the probability weighted characteristic function. KYBERNETIKA, 51(4):571-587, 2015. [p510]

B. C. Monsell. The x-13a-s seasonal adjustment program. In Proceedings of the 2007 Federal Committee On Statistical Methodology Research Conference. URL http://www. fcsm. gov/07papers/Monsell. II-B. pdf, 2007. [p515]

B. C. Monsell, J. A. D. Aston, and S. J. Koopman. Toward x-13? Technical report, United States Census Bureau, 2003. URL https://www. census.gov/content/dam/Census/library/workingpapers/2003/adrm/jsm2003bcm.pdf. [p515]

Y. Mu and X. He. Power transformation toward a linear regression quantile. Journal of the American Statistical Association, 102(477):269-279, 2007. [p510]

P. Newbold and C. W. Granger. Experience with forecasting univariate time series and the combination of forecast. Journal of Royal Statistical Society, 137(2):131-165, 1974. [p531]

M. O'Hara-Wild, R. Hyndman, E. Wang, G. Caceres, T.-G. Hensel, and T. Hyndman. fable: Forecasting Models for Tidy Time Series, 2021a. URL https: //cran. r-project.org/package=fable. R package version 0.3.1. [p529]

M. O'Hara-Wild, R. Hyndman, E. Wang, D. Cook, G. Athanasopoulos, and D. Holt. fabletools: Core Tools for Packages in the 'fable' Framework, 2021b. URL https: //cran. r-project. org/package=fabletools. $\mathrm{R}$ package version 0.3.1. [p529] 
D. R. Osborn, A. P. L. Chui, J. Smith, and C. R. Birchenhall. Seasonality and the order of integration for consumption. Oxford Bulletin of Economics and Statistics, 50(4):361-377, 1988. [p514]

C. C. Pegels. On startup or learning curves: An expanded view. AIIE Transactions, 1(3):216-222, 1969. [p508]

W. M. Persons. Indices of Business Conditions: An Index of General Business Conditions, volume 1. Harvard University Press, 1919. [p515]

B. Pfaff, E. Zivot, and M. Stigler. urca: Unit Root and Cointegration Tests for Time Series Data, 2016. URL https://cran.r-project.org/package=urca. R package version 1.3-0. [p523]

P. C. B. Phillips and P. Perron. Testing for a unit root in time series regression. Biometrika, 75(2):335-346, 1988. [p513]

A. J. Quiroz, M. Nakamura, and F. J. Pérez. Estimation of a multivariate box-cox transformation to elliptical symmetry via the empirical characteristic function. Annals of the Institute of Statistical Mathematics, 48(4):687-709, 1996. [p510]

R Core Team. R: A Language and Environment for Statistical Computing. R Foundation for Statistical Computing, Vienna, Austria, 2016. URL https: //www. R-project. org/. ISBN 3-900051-07-0. [p508, 509, 522]

D. J. Reid. A comparative study of time series prediction techniques on economic data. International Journal of Forecasting, 19(2):303-308, 1969. [p531]

J. A. Ryan, J. M. Ulrich, R. Bennett, and C. Joy. xts: eXtensible Time Series, 2020. URL https: //cran. rproject.org/package=xts. R package version 0.12.1. [p523]

S. E. Said and D. A. Dickey. Testing for unit roots in autoregressive-moving average models of unknown order. Biometrika, 71(3):599-607, 1984. [p513]

R. M. Sakia. The box-cox transformation technique: A review. Journal of the Royal Statistical Society Series D, 41(2):169-178, 1992. [p510]

C. Sax and D. Eddelbuettel. seasonal: R Interface to X-13-ARIMA-SEATS, 2020. URL https://cran. rproject.org/package=seasonal. R package version 1.7.1. [p523]

J. Shiskin. Types of Economic Fluctuations, pages 5-6. NBER, 1957. URL http://www. nber.org/ chapters/c2726. [p515]

J. Shiskin, A. H. Young, and J. C. Musgrave. The $x-11$ variant of the census-ii method seasonal adjustment program. Technical Report 15, Bureau of the U.S. Census, 02 1967. URL https: //www. census.gov/ts/papers/ShiskinYoungMusgrave1967.pdf. [p515]

J. G. Staniswalis, T. A. Severini, and P. G. Moschopoulos. On a data based power transformation for reducing skewness. Journal of Statistical Computation and Simulation, 46(1-2):91-100, 1993. [p510]

A. S. Taylan, H. T. Selamlar, and G. Yapar. ATAforecasting: Automatic Time Series Analysis and Forecasting Using the Ata Method, 2021a. URL https://cran.r-project.org/package=ATAforecasting. R package version 0.0.55. [p508]

A. S. Taylan, H. T. Selamlar, and G. Yapar. fable.ata: 'ATAforecasting' Modelling Interface for 'fable', 2021b. URL https://cran.r-project.org/package=fable.ata. R package version 0.0.2. [p529]

A. Trapletti and K. Hornik. tseries: Time Series Analysis and Computational Finance, 2020. URL https: //CRAN. R-project.org/package=tseries. R package version 0.10-48. [p523]

J. W. Tukey. Exploratory Data Analysis. Pearson, 1977. [p511]

X. Wang, K. A. Smith, and R. J. Hyndman. Characteristic-based clustering for time series data. Data Mining and Knowledge Discovery, 13(3):335-364, 2006. [p514]

J. Whittaker, C. Whitehead, and M. Somers. The neglog transformation and quantile regression for the analysis of a large credit scoring database. Journal of the Royal Statistical Society Series C, 54(4): 863-878, 2005. [p511]

D. Wuertz, T. Setz, and Y. Chalabi. timeSeries: Rmetrics - Financial Time Series Objects, 2020. URL https://cran.r-project.org/package=timeSeries. R package version 3062.100. [p523]

Z. Yang. A modified family of power transformations. Economics Letters, 92(1):14-19, 2005. [p511] 
G. Yapar. Modified simple exponential smoothing. Hacettepe University Journal of Mathematics and Statistics, 47(3):741-754, 2018. [p507, 522, 531]

G. Yapar, I. Yavuz, and H. T. Selamlar. Why and how does exponential smoothing fail? an in depth comparison of Ata-simple and simple exponential smoothing. Turkish Journal of Forecasting, 1(1): 30-39, 2017. [p507, 531]

G. Yapar, S. Capar, H. T. Selamlar, and I. Yavuz. Modified holt's linear trend method. Hacettepe University Journal of Mathematics and Statistics, 47(5):1394-1403, 2018. [p507, 531]

G. Yapar, H. T. Selamlar, S. Capar, and I. Yavuz. ATA method. Hacettepe Journal of Mathematics and Statistics, 48(6):1838-1844, 2019. [p507, 531]

I.-K. Yeo and R. A. Johnson. A new family of power transformations to improve normality or symmetry. Biometrika, 87(4):954-959, 2000. [p510, 511]

T. E. Yilmaz, G. Yapar, and I. Yavuz. Comparison of Ata method and croston based methods on forecasting of intermittent demand. Mugla Journal of Science and Technology, 5(2):49-55, 2019. [p507]

Ali Sabri Taylan

Dokuz Eylul University

The Graduate School of Natural and Applied Sciences

Tinaztepe Campus Buca, Izmir, TURKEY

ORCiD 0000-0001-9514-934X alisabri taylan@gmail .com

Guckan Yapar

Dokuz Eylul University

The Graduate School of Natural and Applied Sciences

Tinaztepe Campus Buca, Izmir, TURKEY

ORCiD 0000-0002-0971-6676

guckan. yapar@deu.edu.tr

Hanife Taylan Selamlar

Dokuz Eylul University

The Graduate School of Natural and Applied Sciences

Tinaztepe Campus Buca, Izmir, TURKEY

ORCiD 0000-0002-4091-884X

hanife. taylan@deu.edu. tr 